\title{
Modeling of Extrusion With Slip Boundary Conditions
}

\author{
H. E. H. MEIJER* and C. P. J. M. VERBRAAK \\ DSM Research \\ Geleen, Holland
}

\begin{abstract}
Slip at boundaries is possible in viscous flows; for instance, in the extrusion of foodstuffs, water-containing materials, and some polymers. There are two phenomenological descriptions of slip: The first is based on the presence of a very thin, low viscosity boundary layer and has been derived for capillary flow and extrusion; the second is based on a Coulomb-friction mechanism and has been derived for capillary flow only. After a survey of these results, the friction model is derived for the extrusion process. All calculations are as simple as possible: twodimensional, Newtonian, isothermal, and with constant boundary layer parameters or coefficients of friction. A strong dependence of pumping characteristics and efficiency on the slip boundary conditions, and also on the extruder length in the case of friction, was found, especially when slip is only allowed for at the screw surface. Exercises like these may help in understanding abnormal extrusion behavior of slippery materials in practice.
\end{abstract}

\section{INTRODUCTION}

$\mathbf{M}$ ost extruders are pumps based on drag flow, and their working principle therefore completely depends on stick of the fluid at the boundaries. Stick is accepted and experimentally demonstrated, but as a phenomenon it is still remarkable. Certainly with polymer melt flows, microscopic stick of molecules at walls would result in a strong degradation; therefore, stick can be better considered as a macroscopic effect only. On a local scale, slip must be present if a fluid leaves a die or a milling roll to prevent infinitely large stresses inside the material. With some materials, real macroscopic slip is demonstrated: foodstuffs like dough and meat; water-containing materials like gypsum, cement, and clay; polymer solutions and pure polymers like poly (vinyl chloride) (PVC) (1-7), ultra-high-molecular weight high-density polyethylene (UHMWHDPE) (8, 9), rubbers (10), bulk-molding compound (BMC), and sheetmolding compound (SMC) (11).

In the modeling of flows with slip, two different kinds of boundary conditions are introduced. The first model gives a velocity-dependent wall shear stress $\tau=\tau(u)$ and is used when a very thin low viscous slip layer is present between fluid and wall, (see Fig. Ib). Pressure flow in capillaries $(12,13)$ and drag and pressure flow in extruders (14-16) have been calculated with this boundary condition. The sec- ond model gives a shear stress that is pressure dependent $\tau=\tau(P)$ and can be used if there is (relative to the cohesive forces) insufficient adhesion between fluid and wall (see Fig. 1c). This model has been only applied to capillary flow (17).

The introduction of a more general boundary condition $\tau=\tau(u, d u / d y, \mathrm{P})$ will only make sense if the simpler extremes are investigated first and if reliable experimental methods are available. [Remember $\tau=\tau(d u / d y)$ is generally valid everywhere in the flow because of the constitutive equation, but is also used to indicate stick, see Fig. 1a.] The same argument holds for the temperature, time, and place dependence $\tau=\tau(T, t, x)$, for example, in the case of diffusion of low-molecular-weight material to the surface or in the case of melting.

A pressure-dependent boundary condition $\tau$

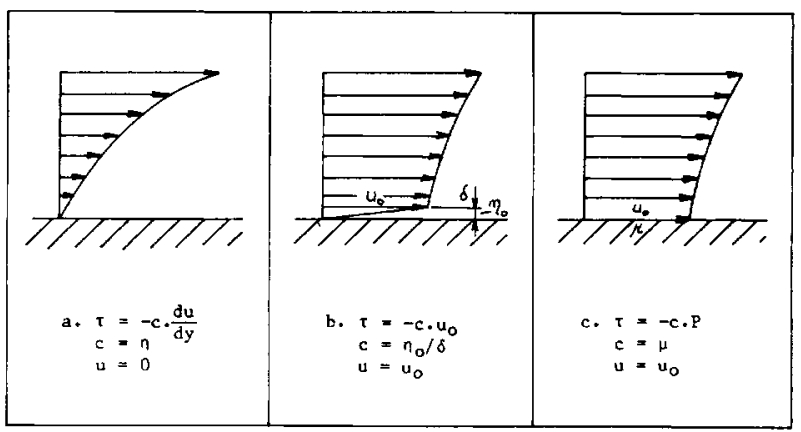

Fig. 1. Different boundary conditions. 
$=\tau(P)$ is normally associated with dry Coulomb friction and applied when two solids slide over each other. This same condition is consequently used in the analysis of the plug flow in the solids-conveying zone of extruders (18). The coefficient of friction $\mu$ is dependent on temperature, velocity, and even pressure $\mu=\mu(T, u, P)$.

Pressure-dependent boundary conditions always result in an exponential increase of pressure (feed section) or force (tire brake, winch). The increase of pressure in the feed section of extruders causes the boundary condition to change from slip $[\tau=\tau(P)]$ to stick $[\tau=\tau(d u / d y)]$ since local melting takes place (19-21). (The heat generated is proportional to the local pressure.) This melting stops the exponential pressure buildup and separates feed section (undeformable plug) and melting section (completely deformable plug) (22). In the slip model with a pressure-dependent condition, derived below for the extrusion process, the change from a deformable plug that slips to one that sticks is smooth and continuous even in the unrealistic most simple isothermal Newtonian case with a constant coefficient of friction $\mu$.

\section{CAPILLARY FLOW}

As an introduction to the slip models, the flow in capillaries will be considered first. In all cases, the momentum equation ( $E q$ l) must be solved combined with the Newtonian constitutive equation $(E q 2)$ and the different boundary conditions (see Fig. 2).

$$
\begin{aligned}
\frac{d P}{d z} & =-\frac{1}{r} \frac{d}{d r}(r \tau) \\
\tau & =-\eta \frac{d w}{d r}
\end{aligned}
$$

Results will be presented in dimensionless form

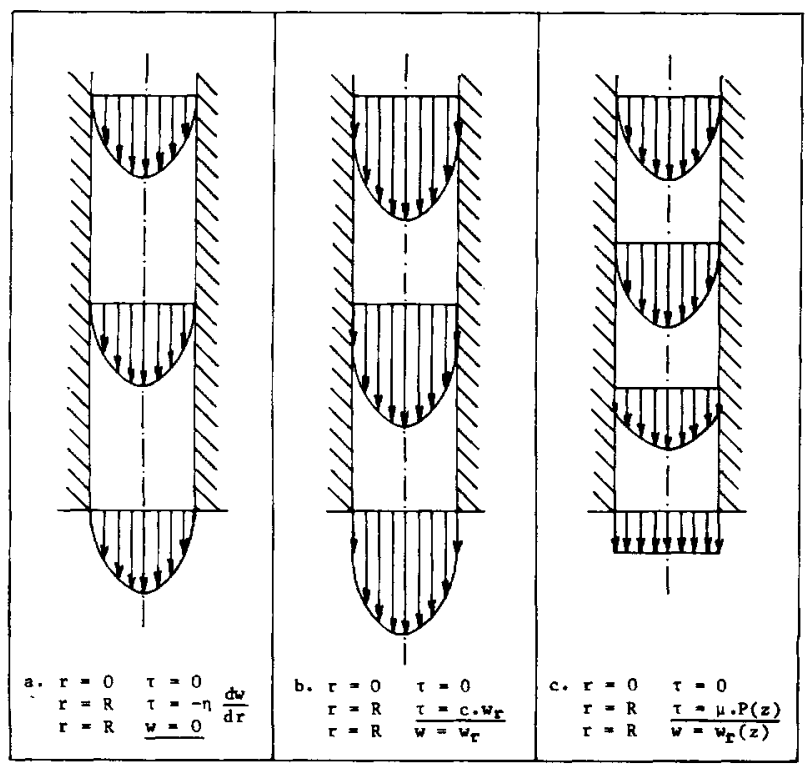

Fig. 2. Capillary flow. with

$$
\begin{aligned}
& \tilde{r}=r / R \tilde{z}=z / R \bar{w}=Q / \pi R^{2} \tilde{w}=w / \bar{w} \\
& \tilde{Q}=Q / \pi R^{2} \bar{w}=1 \tilde{P}=P R / \eta \bar{w}
\end{aligned}
$$

Stick

The Hagen-Poiseuille formula for the throughput reads

$$
\tilde{Q}=-\frac{1}{8} \frac{d \tilde{P}}{d \tilde{z}}
$$

and the velocity profile is given by

$$
\tilde{w}=\frac{1}{4} \frac{d \tilde{P}}{d \tilde{z}}\left(\tilde{r}^{2}-1\right)=2 \tilde{Q}\left(1-\tilde{r}^{2}\right) \text {. }
$$

\section{Boundary Layer Slip}

The Mooney expressions (12) for throughput and velocity profile read

$$
\begin{gathered}
\tilde{Q}=-\frac{1}{8} \frac{d \tilde{P}}{d \tilde{z}}\left(1+\frac{4}{B}\right) \\
\tilde{w}=\frac{1}{4} \frac{d \tilde{P}}{d \tilde{z}}\left(\tilde{r}^{2}-1-\frac{2}{B}\right)
\end{gathered}
$$

with the dimensionless slip coefficient $B=$ $c R / \eta$ (see Fig. $1 b$ ).

In Fig. 3, the velocity profiles are given for different values of $B$. In Fig. 3a, the throughput is kept constant $\tilde{Q}=1$; in Fig. $3 b$, the pressure gradient is kept constant $d \tilde{P} / d \tilde{z}=-8$. In Fig. $4 a$, the pressure gradient as a function of slip coefficient $B$ is plotted with constant throughput; in Fig. $4 b$, the throughput is given, now keeping the pressure gradient constant.

\section{Friction Slip}

The analysis of friction slip $(8,9)$ is somewhat more complicated because the shear stress at the wall is function of the local pressure and consequently of $z$. Moreover, a stick-slip transition point is present. The fluid sticks to the wall until, with decreasing pressure, the shear stress on the boundary becomes smaller than the shear stress needed to maintain stick (Fig. 2c).

It is convenient to start with $z=0$ at the end of the capillary. Furthermore, a nonzero end pressure, $P_{o}$ is defined there. The pressure at the beginning of the capillary is $P_{L}$. In the slip area, the pressure and the velocity profile read

$$
\begin{aligned}
\tilde{P} & =\tilde{P}_{o} e^{2 \mu \tilde{z}} \\
\tilde{w} & =\tilde{G}+1 / 4 \mu \tilde{P}_{o} e^{2 \mu \tilde{z}}\left(2 \tilde{r}^{2}-1\right)
\end{aligned}
$$

At the transition point the shear stresses for slip and stick are the same; therefore,

$$
\begin{aligned}
& \tilde{P}_{1}=-\frac{4 \tilde{Q}}{\mu} \\
& \tilde{z}_{1}=\frac{1}{2 \mu} \ln \left(\frac{-4 \tilde{Q}}{\mu \tilde{P}_{o}}\right)
\end{aligned}
$$



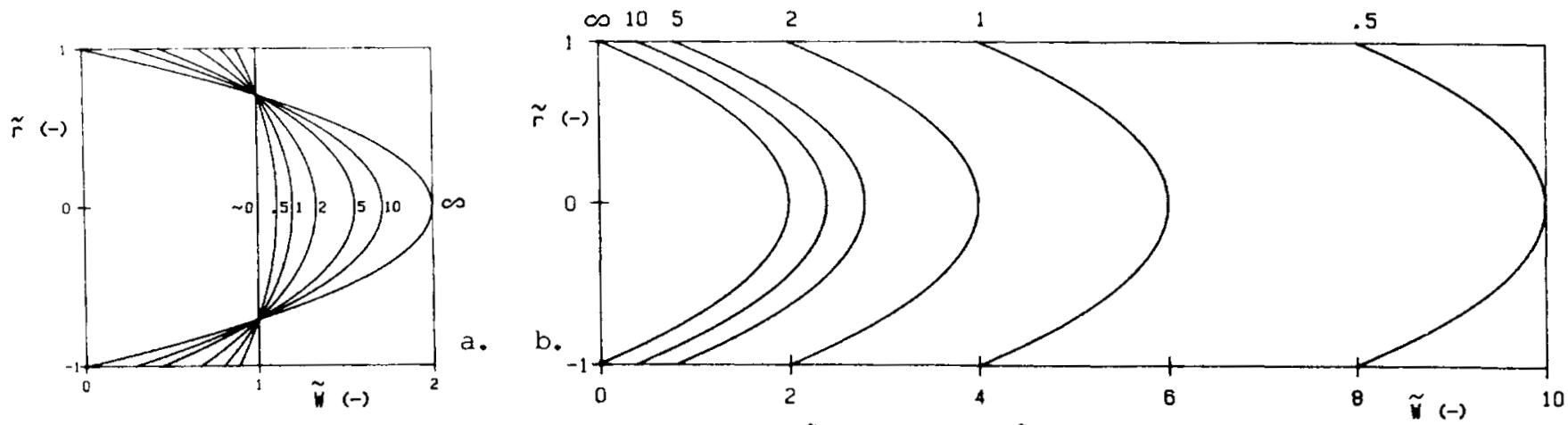

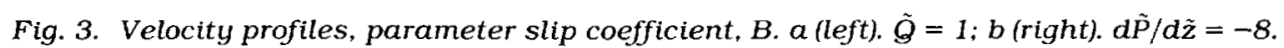

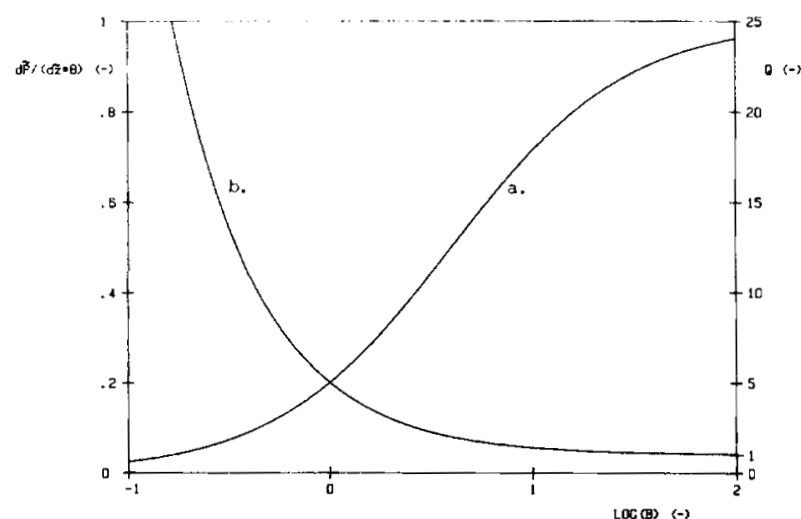

Fig. 4. Pressure gradient and throughput us. slip coefficient. a. $\tilde{Q}=1 ; b . d \tilde{P} / d \tilde{z}=-8$.

Consequently, the (linear) pressure profile in the stick area reads

$$
\tilde{P}=\tilde{P}_{1}+\frac{d \tilde{P}}{d \tilde{z}}\left(\tilde{z}-\tilde{Z}_{1}\right),
$$

with $\frac{d \tilde{P}}{d \tilde{z}}$ given in Eq. 4. The pressure at the beginning of the capillary is

$$
\tilde{P}_{L}=-4 \tilde{Q}\left(2 \tilde{L}+\frac{1}{\mu}\left(1-\ln \left(\frac{-4 \tilde{Q}}{\mu \tilde{P}_{o}}\right)\right)\right) \text {. }
$$

In Fig. 5, the pressure profiles over the capillary are shown for different values of the coefficient of friction. Figure 6 shows the continuously changing velocity profiles in the slip region (compare with Fig. 3).

\section{EXTRUSION}

To investigate extrusion behavior with slip, the most simple two-dimensional model of a drag pump will be used (see Fig. 7). The agreement with real extruders is more easily seen if, in the latter, the screw is thought to be stationary and the barrel rotating in opposite direction; furthermore, the screw should be thought of as unrolled to a flat U-shaped profile over which an infinite flat plate is moving. Finally, only the component of the barrel velocity in channel direction should be considered and the motion perpendicular to the flights neglected. The equation of motion ( $E q 14)$ and the Newtonian

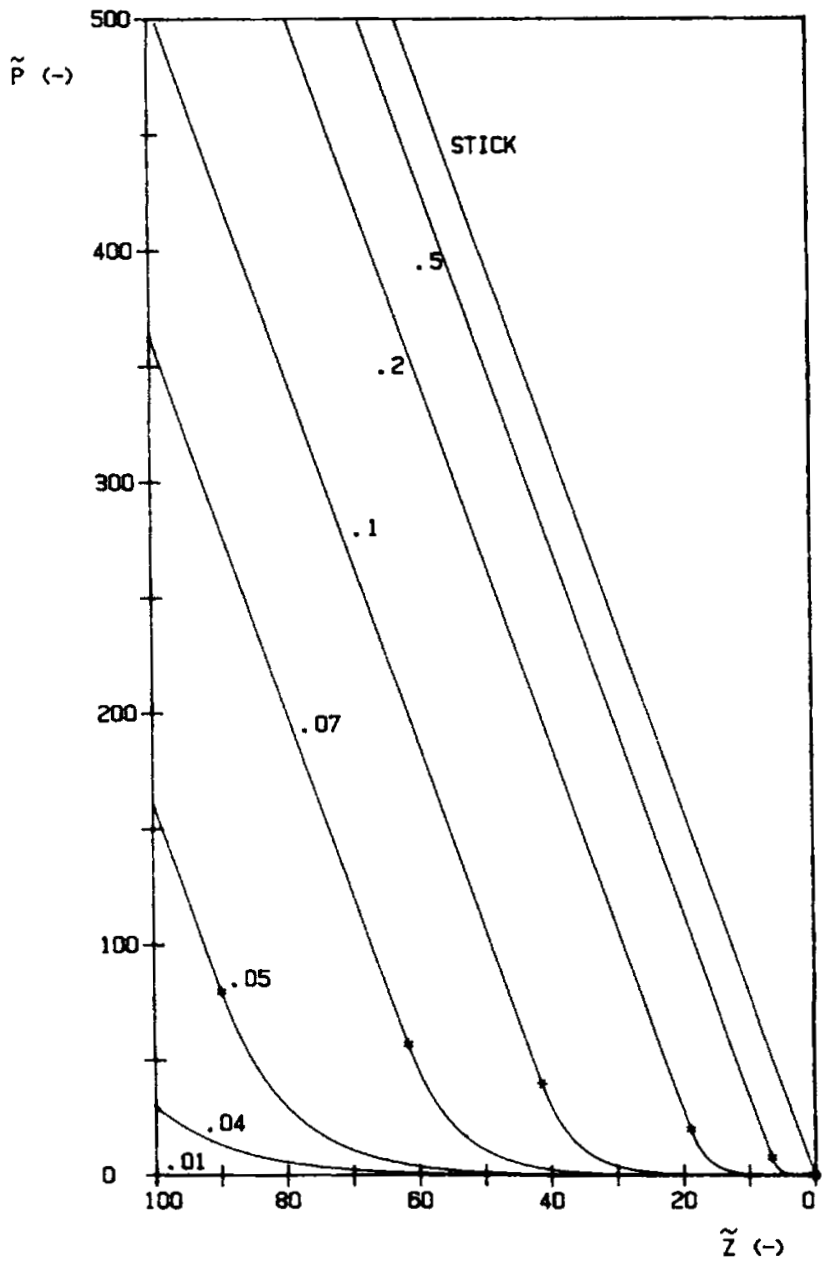

Fig. 5. Pressure profiles over a capillary, parameter friction coefficient, $\mu$. Stick-slip transition points are denoted with " $\left[R=0.004 \mathrm{~m}, \eta=1000\right.$ Pa.s, $P_{\circ}=100 \mathrm{~Pa}$ (small!], $w=-0.04 \mathrm{~m} / \mathrm{s}, \tilde{Q}=11$.

constitutive equation ( $E q 15)$ now read

$$
\begin{aligned}
\frac{d P}{d x} & =-\frac{d \tau}{d y} \\
\tau & =-\eta \frac{d u}{d y}
\end{aligned}
$$

Because there is no symmetry in this extruder, we have to deal with two distinguishable boundaries, $y=O$ (screw surface) and $y=H$ (barrel surface), where slip can occur. This 


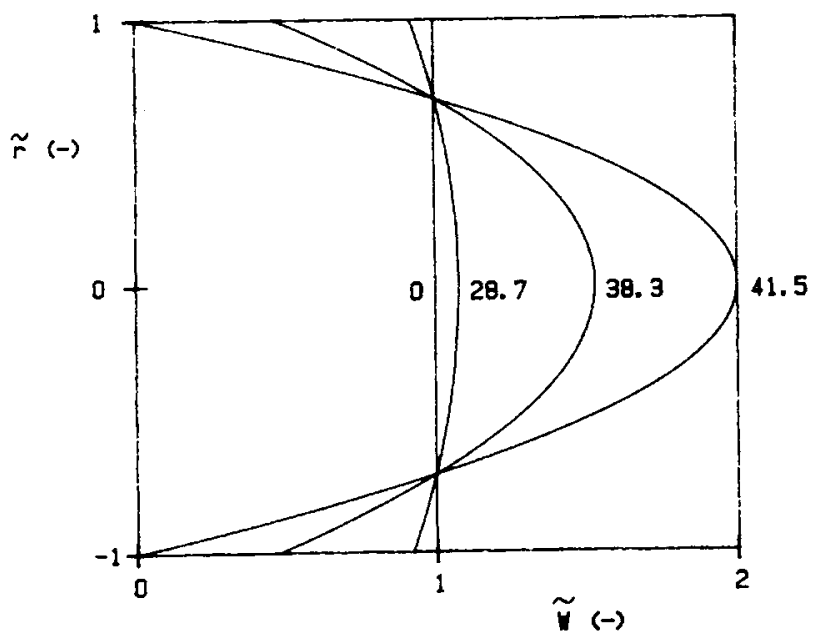

Fig. 6. Developing velocity profile $(\mu=0.1)$, parameter axial distance, $\tilde{z}$.

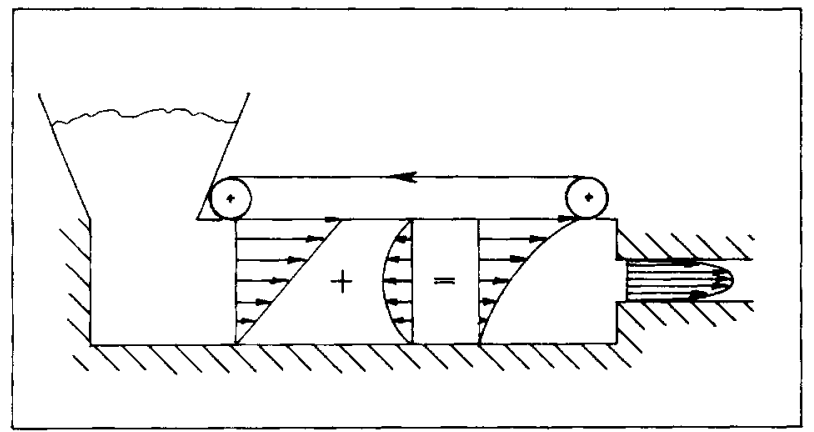

Fig. 7. Extruder model.

yields some more complicated situations than in the case of capillary flow. Again, dimensionless expressions will be used:

$$
\begin{aligned}
& \tilde{x}=x / H \quad \tilde{y}=y / H \quad \tilde{u}=u / U \\
& \tilde{Q}=Q / 1 / 2 U H \quad K=\frac{d \tilde{P}}{d \tilde{x}}=\frac{d P}{d x} H^{2} / \eta U \\
& \tilde{P}=P H / \eta U
\end{aligned}
$$

\section{Stick}

With use of the throttle ratio, $a$, which gives the ratio of pressure flow and draw flow,

$$
a=\frac{\frac{1}{12 \eta} \frac{d P}{d x} H^{3}}{1 / 2 U H}=\frac{H^{2}}{6 \eta U} \frac{d P}{d x},
$$

the expressions for the velocity profile, the throughput, and the pumping efficiency read

$$
\begin{aligned}
\tilde{u} & =\tilde{y}+3 a\left(\tilde{y}^{2}-\tilde{y}\right) \\
\tilde{Q} & =1-a \\
\eta_{p} & =3 a(1-a) /(1+3 a)
\end{aligned}
$$

with $\eta_{p}$ defined as

$$
\eta_{p}=\frac{Q \cdot \Delta P}{E}
$$

with $E=-U \int_{0}^{L} \tau_{y=H} d x$ and $\Delta P=\int_{0}^{L}(d P / d x) d x$
$=P_{e}-P_{o}$. (This definition makes the efficiency zero if either $Q=0$ or $\Delta P=0$; therefore, it is for a real pump.)

Figure 8 shows the velocity profiles for different values of $a$. If $a<0$ the pump is overfed; negative pressure gradients are needed for a volume flow that is larger than the drag flow only (compare $E q 19$ ). The efficiency is given in Fig. 9 showing the well-known maximum value $\eta_{p}=1 / 3$ for $a=1 / 3$.

\section{Boundary Layer Slip}

\section{Slip at Both Boundaries}

Calculations of flow in extruders with slip boundary conditions were given by Schlegel (23), Worth, et al. $(24,25)$, Mennig $(26,27)$, and Perwadtschuk (28). However, the first straightforward analysis of the boundary layer slip model was given by Holslag (14) and Ingen Housz (15) in 1980 (not published until 1983 in Ref. 16). The boundary conditions for this problem read

$$
\begin{array}{lll}
y=o & u=u_{o} & \tau=-c_{o} u_{o} \\
y=H & u=u_{h}=U-u_{s} & \tau=-c_{h} u_{s}
\end{array}
$$

Again, the dimensionless slip coefficients, $B_{o}=$ $c_{0} H / \eta$ and $B h=c_{h} H / \eta$ are used.

Solving the momentum equation $(E q 14)$ with Eqs 15 and 16 and these boundary conditions yields the following expressions for the velocity

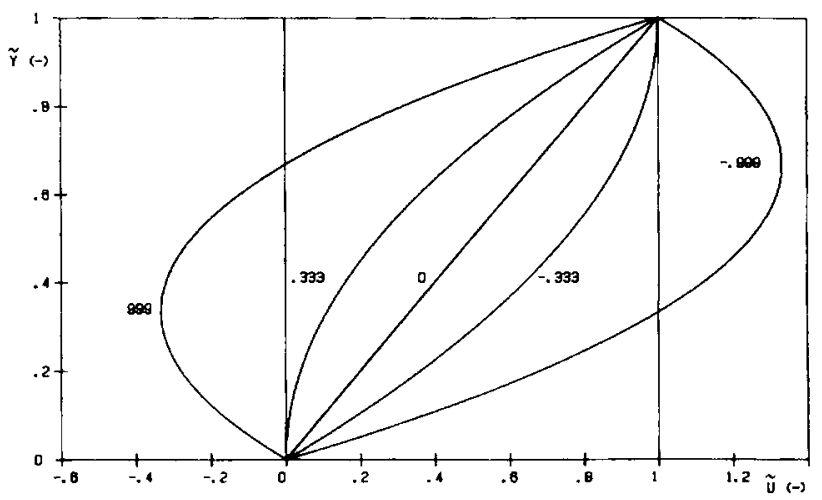

Fig. 8. Velocity profiles, parameter throttle ratio, a.

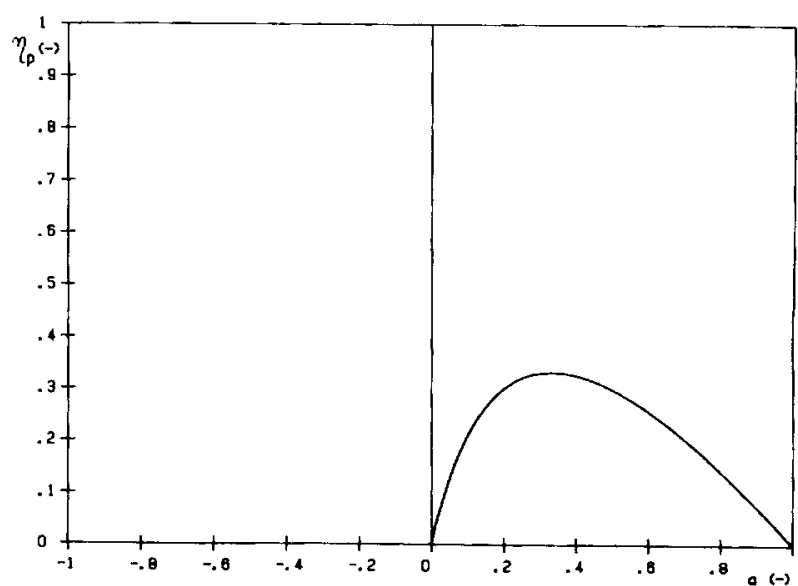

Fig. 9. Pumping efficiency vs, throughput. 
profile, output, and efficiency:

$$
\tilde{u}=1-\tilde{u}_{s}+1 / 2 K\left(\tilde{y}^{2}-1\right)+B_{o} \tilde{u}_{o}(\tilde{y}-1)
$$

with $\tilde{u}_{\mathrm{s}}=\left(B_{o}+K+1 / 2 B_{o} K\right) /\left(B_{h}+B_{o}+B_{o} B_{h}\right)$ and $\tilde{u}_{o}=\left(B_{h}-K-1 / 2 B_{h} K\right) /\left(B_{h}+B_{o}+B_{o} B_{h}\right)$.

$$
\tilde{Q}=1-A
$$

with $A=\left(\left(4+B_{o}+4 B_{o} / B_{h}+12 / B_{h}\right) K / 6-1+\right.$ $\left.B_{o} / B_{h}\right) /\left(1+B_{o}+B_{o} / B_{h}\right)$.

$\begin{aligned} \eta_{p}=((2+ & \left.B_{\mathrm{o}}\right) K-\left(4+B_{o}+4 B_{o} / B_{h}\right. \\ & \left.\left.+12 / B_{h}\right) K^{2} / 6\right) /\left(2 B_{o}+\left(2+B_{o}\right) K\right)\end{aligned}$

For convenience $\mathrm{Eq} 24$ is written in the same form as $E q 19$; however, $a$ and $A$ are not the same, mainly because $A$ can vary from $-1 \leqslant A$ $\leqslant 1$ (letting $0 \leqslant \tilde{Q} \leqslant 2$ or $0 \leqslant Q \leqslant U H$ ) while, without overf eeding, the normal range of $a$ is 0 $\leqslant a \leqslant 1$ (letting $0 \leqslant Q \leqslant 1$ or $0 \leqslant Q \leqslant 1 / 2 U H$ ). This is because when full slip is allowed at the screw surface and slip is prevented at the barrel wall, the maximum transport capacity of the drag flow equals $\tilde{Q}=2$ or $Q=U H$, as will be demonstrated in Slip at the Screw Only, below.

In Fig. 10 some velocity profiles are plotted. Fig. 10a shows the effect of output, keeping the slip coefficients constant. Fig. $10 b$ varies the slip coefficients keeping the output constant. Figure 11 gives the pump characteristics, output $\tilde{Q}$ vs. pressure gradient $K$, varying the slip coefficients. Fig. 12 shows the efficiency as a
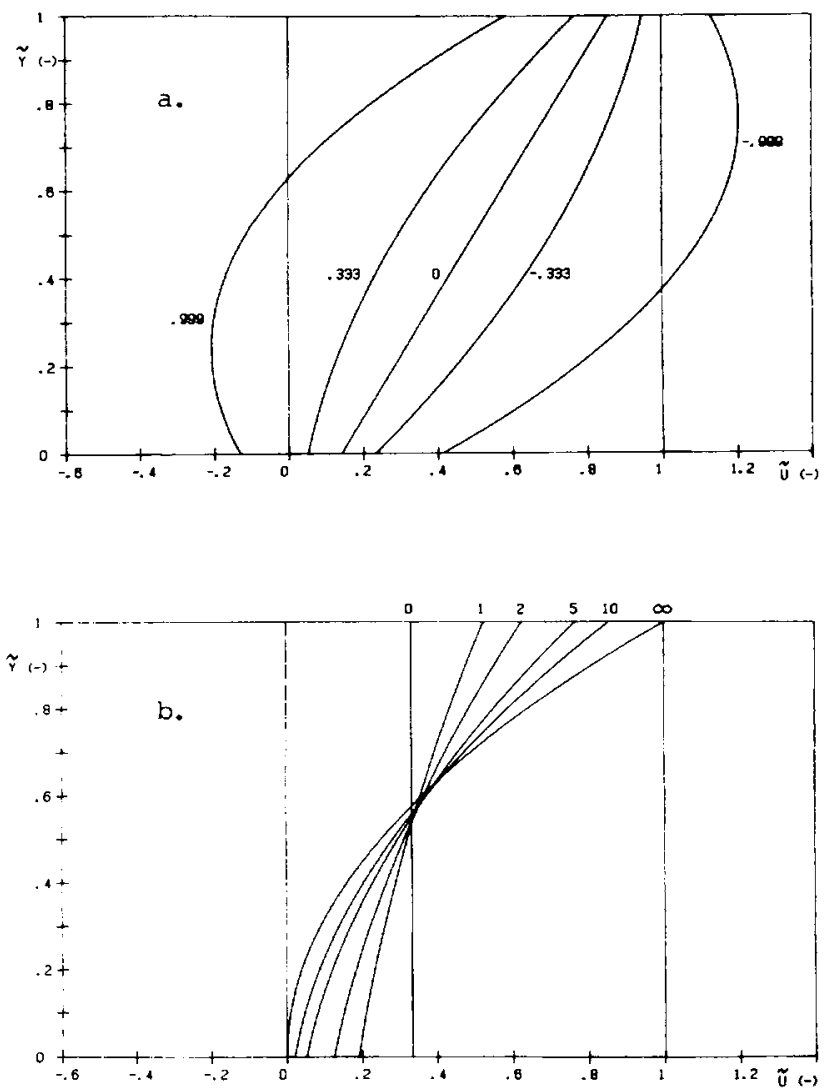

Fig. 10. Velocity profiles with slip at barrel and screw: a. parameter throughput $A\left(B_{o}=B_{h}=5\right)$; $b$. parameter slip coefficient $B_{o}=B_{h}(A=1 / 3)$.

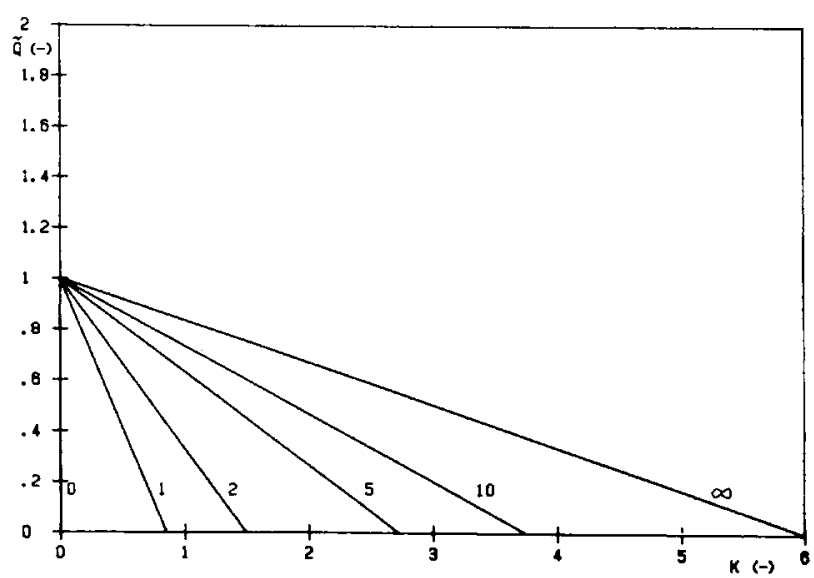

Fig. 11. Pumping characteristics, parameter slip coefficients, $B_{o}=B_{h}$.
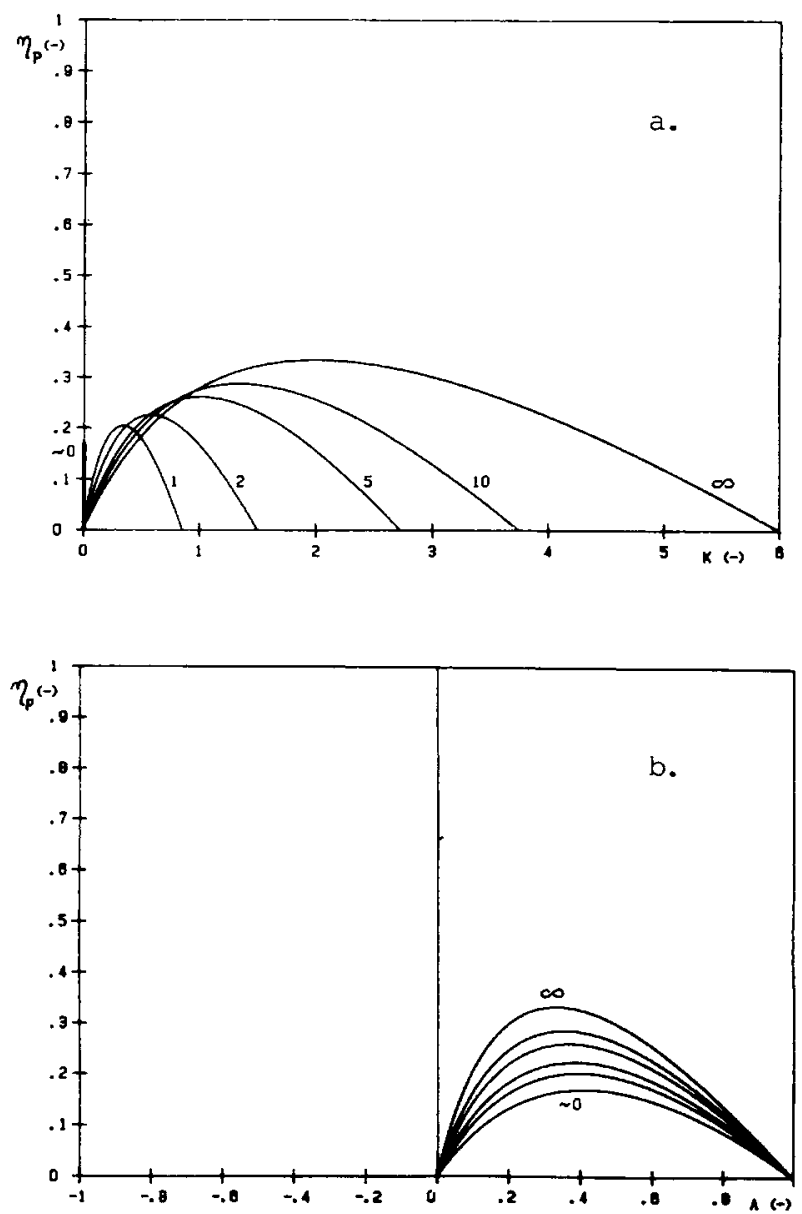

Fig. 12. Pumping efficiency, parameter slip coefficients $B_{o}=B_{h}: a$. vs. pressure gradient; $b$. vs. throughput.

function of pressure gradient (Fig. 12a) and output (Fig. 12b), again with the slip coefficients as parameter.

\section{Slip at the Screw Only}

As can be recognized from Figs. 11 and $12 a$, the pressure generating capacity of the extruder as a pump decreases drastically with increasing slip. Of course one wants to prevent this in practice; attempts are therefore made to prevent the material from slipping at the barrel 
surface; for instance, by making the barrel wall grooved over its total length in axial direction or by putting pins through the barrel wall. Grooves and pins should break through slip layers and lead the forces from the barrel directly into the material. By taking $B_{h} \rightarrow \infty$ (therefore, $u_{s} \rightarrow o, u_{h} \rightarrow U$ at $y=H$ ), Eqs 23, 24, and 25 change into

$$
\tilde{u}=1+1 / 2 K\left(\tilde{y}^{2}-1\right)+B_{o} \tilde{u}_{o}(\tilde{y}-1)
$$

with $\tilde{u}_{o}=(1-1 / 2 K) /\left(1+B_{o}\right)$;

$$
\tilde{Q}=1-A
$$

with $A=\left(\left(4+B_{o}\right) K / 6-1\right) /\left(1+B_{o}\right)$;

$$
\begin{aligned}
& \eta_{p}=\left(\left(2+B_{o}\right) K-\left(4+B_{o}\right) K^{2} / 6\right) / \\
& \cdot\left(2 B_{o}+\left(2+B_{o}\right) K\right) .
\end{aligned}
$$

The interesting consequences of these measures can be seen directly from the corresponding Fig. 13, the velocity profiles; Fig. 14, the pump characteristics; and Fig. 15, the efficiency. Maximum output of the drag flow is increased by a factor of two, and pumping eff $\mathrm{i}-$ ciency has overcome its natural maximum of $33.3 \%$ and can be increased to almost $100 \%$, as is the case with the disk pack, which has two moving walls $(29,30)$. Of course, this only holds if extreme slip is possible at the screw surface and if the required pressure gradients are small (the driving power still comes from one side only in contrast to the disk pack).
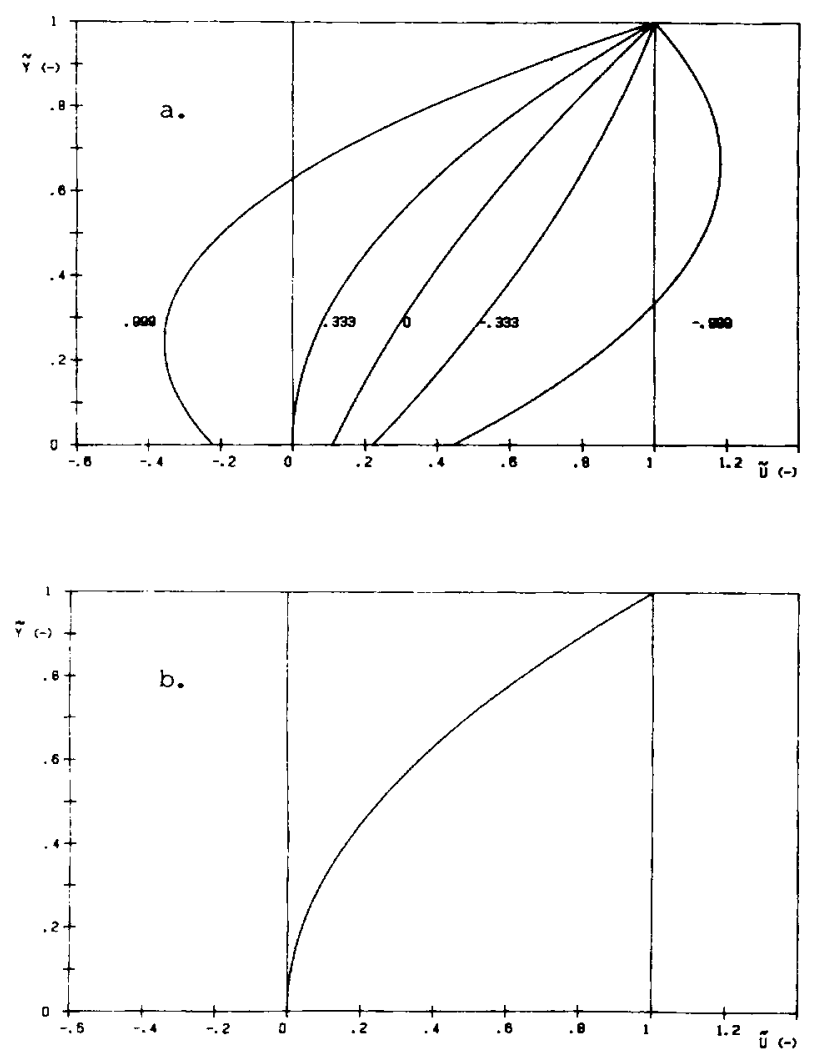

Fig. 13. Velocity profiles with slip at the screw only: $a$. parameter throughput, $A\left(B_{0}=5\right) ; b$. for all values of $B_{0}$ $(A=1 / 3)$.

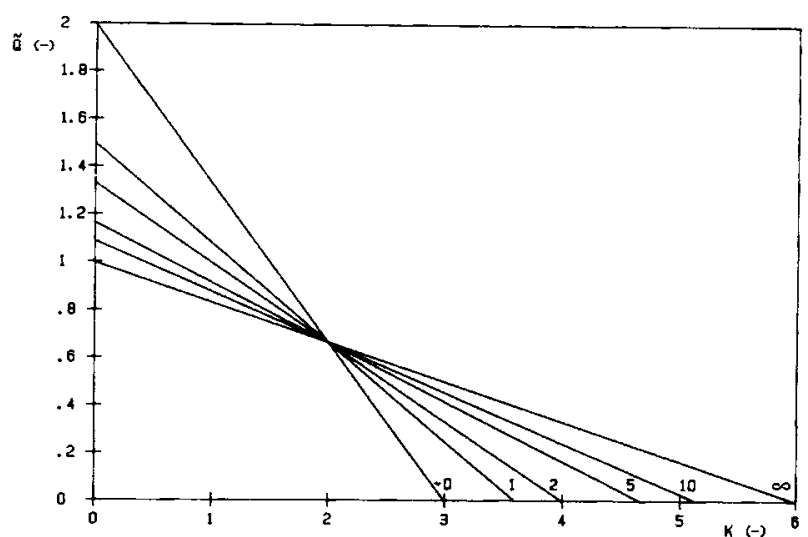

Fig. 14. Pumping characteristics, parameter slip coeffi cient at the screw, $B_{\text {o. }}$
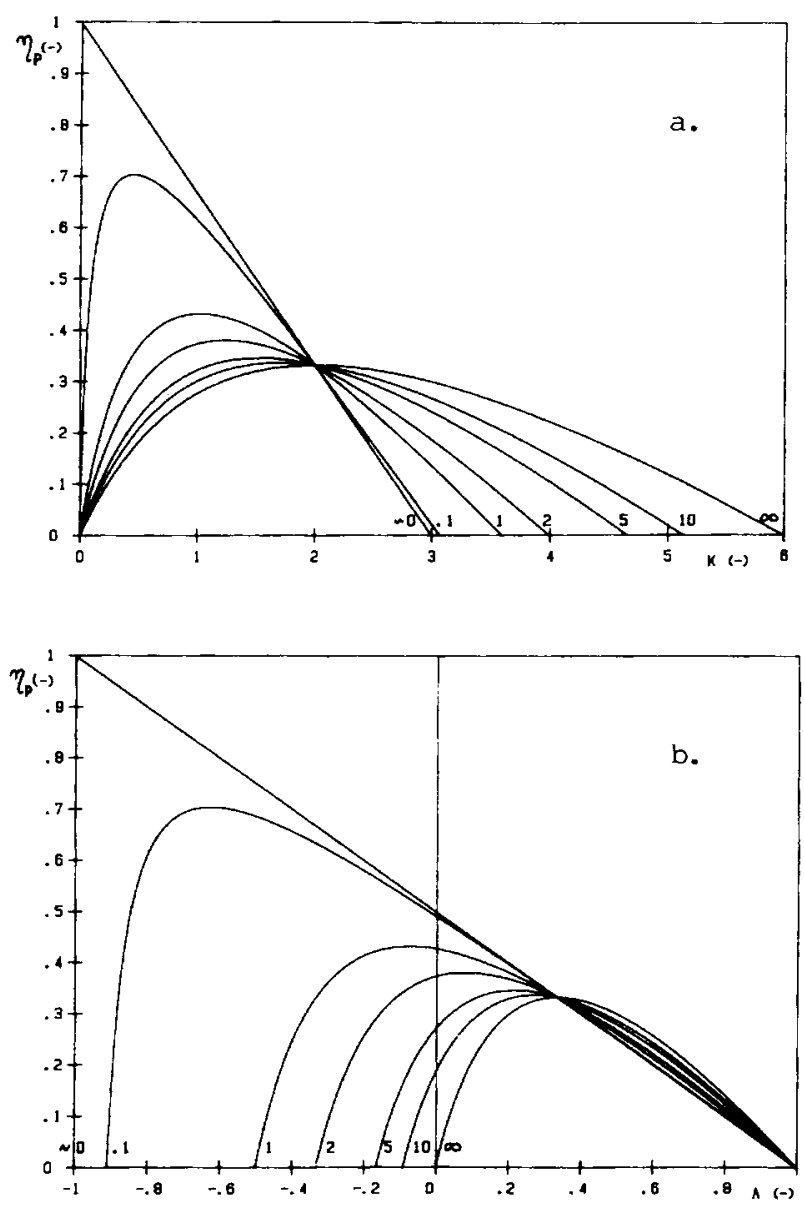

Fig. 15. Pumping efficiency. parameter slip coefficient at the screw, $B_{o}$ : $a$. vs. pressure gradient; $b$. vs. throughput.

\section{Friction Slip}

\section{Slip at Both Boundaries}

As is shown in the analysis of friction slip in capillary flow, the velocity profile and pressure gradient continuously change because of the changing boundary condition $\tau=\tau(P)$. In extruders, because two independent boundaries are present, four situations are possible, as illustrated in Fig. 16:

1. Slip on both sides, which is the starting condition at the beginning of the extruder.

2. Slip at the barrel, stick at the screw. 
H. E. H. Meijer and C. P. J. M. Verbraak

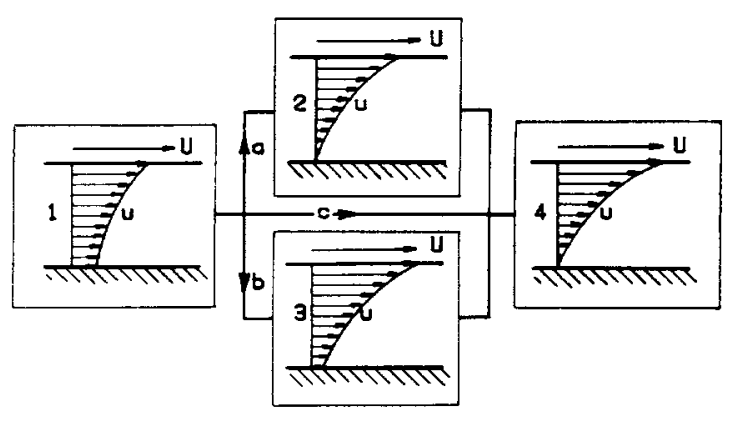

\begin{tabular}{|c|c|c|c|c|}
\hline Situation & 1 & 2 & 3 & 4 \\
\hline$y=0$ & $\begin{array}{l}u=u_{0} \\
\tau=-u_{0} P\end{array}$ & $\begin{array}{l}u=0 \\
T=-\pi \frac{d u}{d y}\end{array}$ & $\begin{array}{l}u=u_{0} \\
T=-\mu_{0} P\end{array}$ & $\begin{array}{l}u=0 \\
y=-n \frac{d u}{d y}\end{array}$ \\
\hline$y=H$ & $\begin{aligned} u & =u_{h} \\
\tau & =-w_{h} P\end{aligned}$ & $\begin{array}{l}u=u_{h} \\
\tau=-u_{h} . P\end{array}$ & $\begin{aligned} u & =U \\
\tau & =-\eta \frac{d u}{d y}\end{aligned}$ & $\begin{array}{l}u=U \\
\tau=-n \frac{d u}{d y}\end{array}$ \\
\hline $\begin{array}{l}x=0 \\
x=x_{1} \\
x=x_{2}\end{array}$ & $P=P_{0}$ & $P=P_{1}$ & $\mathrm{P}=\mathrm{P}_{1}$ & $P=P_{2}$ \\
\hline
\end{tabular}

Fig. 16. The four situations and boundary conditions in friction slip.

3. Stick at the barrel, slip at the screw.

4. Stick on both sides.

Because the extruder generates pressure, the original slip situation 1 will slowly change into the stick situation 4 via situation 2 (route a), 3 (route b), or directly (route c). This is determined by the throughput and, consequently, by the die and operating conditions (the throughput of an extruder-die combination is found as the point of intersection of pump characteristic and die characteristic). It will be shown that for the larger throughputs $(-1<A \leqslant 0)$, the second slipstick transition point from situation 3 to 4 does not exist; therefore, the situation of stick on both sides is never reached.

Solving the momentum equation ( $E q 14)$ with Eqs 15 and 16 and the boundary condition in Fig. 16 yields expressions for the pressure profile and the velocity profile for each situation:

1. $\tilde{P}=\tilde{P}_{o} e^{\Delta \mu \tilde{x}}$ with $\Delta \mu=\mu_{h}-\mu_{o}$

$$
\begin{aligned}
\tilde{u}=1 / 2 & (1-A)+\tilde{P}_{o}\left(\Delta \mu \tilde{y}^{2} / 2+\mu_{o} \tilde{y}\right. \\
& \left.-\mu_{o} / 3-\mu_{h} / 6\right) e^{\Delta \mu \tilde{x}}
\end{aligned}
$$

2. $\tilde{P}=\left(\tilde{P}_{1}-(1-A) / \mu_{h}\right) e^{3 / 2 \mu_{h}\left(\tilde{x}-\tilde{x}_{1}\right)}$

$$
\begin{gathered}
+(1-A) / \mu_{h} \\
\tilde{u}=(1-A) \tilde{y}+\left(\mu_{h} \tilde{P}_{1}-(1-A)\right) \\
\cdot\left(3 / 4 \tilde{y}^{2}-1 / 2 \tilde{y}\right) e^{3 / 2 \mu_{h}\left(\tilde{x}-\tilde{x}_{1}\right)}
\end{gathered}
$$

3. $\tilde{P}=\left(\tilde{P}_{1}-(1+A) / \mu_{o}\right) e^{-3 / 2 \mu_{0}\left(\tilde{x}-\tilde{x}_{1}\right)}$

$$
+(1+A) / \mu_{o}
$$

$$
\begin{aligned}
\tilde{u}=1 & +(1+A)(\tilde{y}-1) \\
& +\left(\mu_{o} \tilde{P}_{1}-(1+A)\right) \\
& \cdot\left(\tilde{y}-3 / 4 \tilde{y}^{2}-1 / 4\right) e^{-3 / 2 \mu_{0}\left(\tilde{x}-\tilde{x}_{1}\right)}
\end{aligned}
$$

4. $\tilde{P}=\tilde{P}_{2}+6 A\left(\tilde{x}-\tilde{x}_{2}\right)$

$$
\tilde{u}=\tilde{y}+3 A\left(\tilde{y}^{2}-\tilde{y}\right)
$$

With these expressions, the slip-stick transition points can be calculated because at these points the stresses at the boundaries calculated for the different situations are equal. Whether stick first occurs at the screw (route a), at the barrel (route b), or at screw and barrel together (route c) can be determined by calculating the first transition point for all routes and investigating which one is the smallest.

In general there are two-slip stick transition points $x_{1}$ and $x_{2}$. The total length of the extruder is denoted as $x_{e}$ with pressure $P_{e}$.

For route $a$, the first transition point is stick at the screw:

$$
\begin{aligned}
& \tilde{x}_{1}=\ln \left(3(1-A) /\left(\tilde{P}_{o}\left(2 \mu_{o}+\mu_{h}\right)\right)\right) / \Delta \mu \\
& \tilde{P}_{1}=3(1-A) /\left(2 \mu_{o}+\mu_{h}\right) .
\end{aligned}
$$

The second transition point is stick at screw and barrel:

$$
\begin{aligned}
\tilde{x}_{2}= & \tilde{x}_{1}+2 \ln \left(2 A\left(2 \mu_{o}+\mu_{h}\right) /\right. \\
& \cdot((1-A) \Delta \mu)) /\left(3 \mu_{h}\right) \\
\tilde{P}_{2}= & (1+3 A) / \mu_{h} .
\end{aligned}
$$

For route $b$, the first transition point is stick at the barrel:

$$
\begin{aligned}
& \tilde{x}_{1}=\ln \left(3(1+A) /\left(\tilde{P}_{o}\left(2 \mu_{h}+\mu_{o}\right)\right)\right) / \Delta \mu \\
& \tilde{P}_{1}=3(1+A) /\left(2 \mu_{h}+\mu_{o}\right)
\end{aligned}
$$

The second transition point is stick at barrel and screw:

$$
\begin{aligned}
& \tilde{x}_{2}=\tilde{x}_{1}-2 \ln \left(2 A\left(2 \mu_{h}+\mu_{o}\right) /((1+A) \Delta \mu)\right) /\left(3 \mu_{o}\right) \\
& \tilde{P}_{2}=(1-3 A) / \mu_{o} .
\end{aligned}
$$

For route $c$, the only transition point is stick at screw and barrel:

$$
\begin{aligned}
& \tilde{x}_{1}=\ln \left(6 A /\left(\tilde{P}_{\circ} \Delta \mu\right)\right) / \Delta \mu \\
& \tilde{P}_{1}=6 A / \Delta \mu
\end{aligned}
$$

Moreover, an extra condition exists for route c:

$$
\mu_{h} / \mu_{o}=(1+3 A) /(1-3 A) .
$$

From $E q 47$, it is found that when both friction coefficients are given, only one real throughput $\tilde{Q}=1-A$ can be found for which this condition holds. Since route c proves to be rather characteristic (see Fig. 18), a graphical representation of $\mathrm{Eq} 47$ is first given in Fig. 17. For all combinations of friction coefficients, the value of $A$ is found to be between zero and one third.

With Eqs 37, 39, 41, 43, and 45 the complete working area of extruders with friction slip 


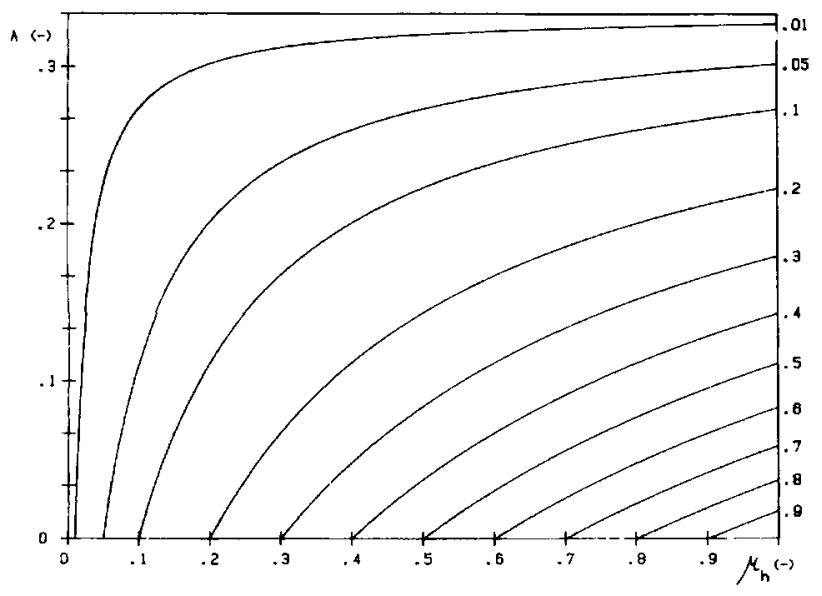

Fig. 17. Throughput, for which the slip-stick transition points at the barrel and the screw coincide, us. barrel friction coefficient. Parameter screw friction coefficient, $\mu_{0}$.

boundary conditions can be constructed. In Fig. 18 an example is given with $\mu_{o}=0.1$ and $\mu_{h}=$ 0.2 (these values are valid for all the following figures). The choice of the values of the friction coefficients is arbitrary in this case. The only condition that should be fulfilled to achieve positive pressure gradients is $\mu_{h}>\mu_{o}$ (see $E q 29$ ).

The same holds for the Darnell and Mol theory: If this theory is applied to an unrolled screw channel and force balances are applied in two perpendicular directions, only positive pressure gradients are achieved if the coefficient of friction at the barrel is larger than the one at the screw. This is why Darnell and Mol took their remarkable combination of one force and one momentum balance!

Figure 18 shows the slip-stick transition points $\tilde{x}_{1}$ and $\tilde{x}_{2}$ and consequently the four different situations in between as a function of the parameter $A$, which stands for the throughput, $\tilde{Q}=1-A$. Extrusion follows vertical lines in this plot starting at $\tilde{\boldsymbol{x}}=0$ at the given throughput $A$. Right from route c, route a is found with stick at the screw first. Left from route $\mathrm{c}$, route $\mathrm{b}$ is found with first stick at the barrel. If $A \leqslant 0$, the second transition point is never reached (route $b^{\prime}$ ). The throughput is too large and the material must always slip at the screw, as is illustrated in Fig. 19, which shows the developing velocity profiles for some given routes, characterized by the throughput $A$.

The pressure profiles are also interesting. Figure 20 shows the pressure $\tilde{P}$ as a function of distance $\tilde{x}$, again with the throughput parameter $A$. Slip-stick transition points and, consequently, change in pressure generating capacity are denoted with a star. For $A>0$ the stickstick situation with the linear pressure profile is always reached. For $A \leqslant 0$ (route $b^{\prime}$ ) this is not the case.

If the total extruder length, $x_{e}$, is fixed, the pump characteristic $(P-Q$ relation) can be calculated (see Fig. 21). With decreasing extruder length, the influence of the slip area is

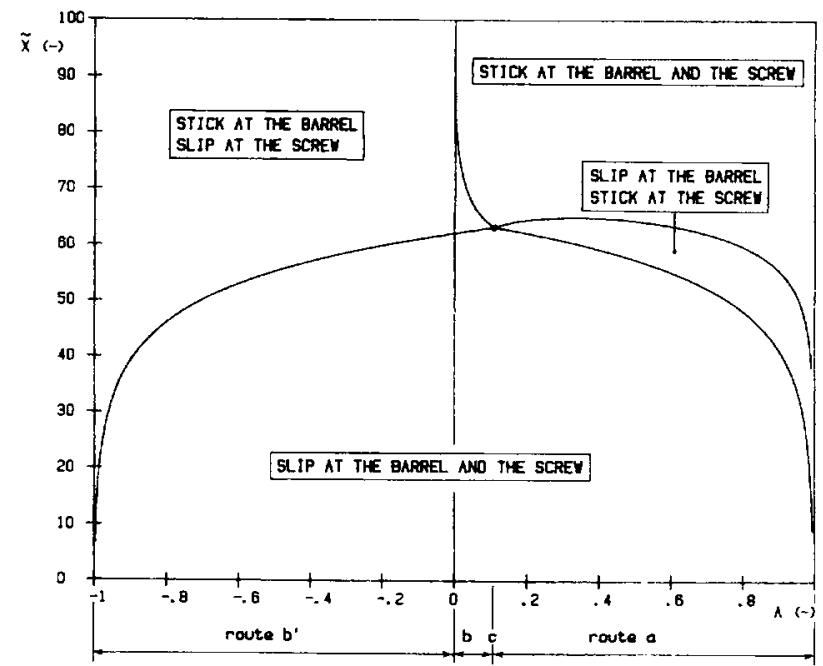

Fig. 18. Working area for extruders with friction slip at barrel and screw $\left(H=0.006 \mathrm{~m}, \eta=1000\right.$ Pa.s, $P_{o}=1000$ $\mathrm{Pa}, U=0.5 \mathrm{~m} / \mathrm{s}, \mu_{o}=0.1, \mu_{h}=0.2$ ).

larger and the pump characteristic more nonlinear. The interesting increase in $Q_{\max }$, first found with one side boundary layer slip (Fig. 14) is again demonstrated. The pumping efficiency also can be computed; however, because of the continuously changing shear stress at the barrel wall, we must now integrate over $x$ :

$$
\begin{aligned}
\eta_{p}=1 / 2 & (1-A)\left(\tilde{P}_{e}-\tilde{P}_{o}\right) / \\
& \left(\int 1+\int 2 \text { or } 3+\int 4\right)
\end{aligned}
$$

with

$$
\begin{aligned}
& \int_{1}=\mu_{h} \tilde{P}_{o}\left(e^{\Delta \mu \tilde{x}_{1}}-1\right) / \Delta \mu \\
& \int_{2}=2\left(\mu_{h} \tilde{P}_{1}-(1-A)\right)\left(e^{3 / 2 \mu_{h}\left(\tilde{x}_{2}-\tilde{x}_{1}\right)}-1\right) /\left(3 \mu_{h}\right) \\
& \quad+(1-A)\left(\tilde{x}_{2}-\tilde{x}_{1}\right)
\end{aligned}
$$

An illustration of the pumping efficiency of extruders with different length, $\tilde{x}_{e}$, is given in Fig. 22. Again high efficiencies are found if slip is present in a substantial part of the extruder and if the pressure gradients required are not too large: Consequently, high throughputs result especially when $A \leqslant 0$.

\section{Slip at the Screw Only}

To give a more pronounced effect, the friction at the barrel is chosen to be much larger than that of the screw in the previous figures. Ex- 

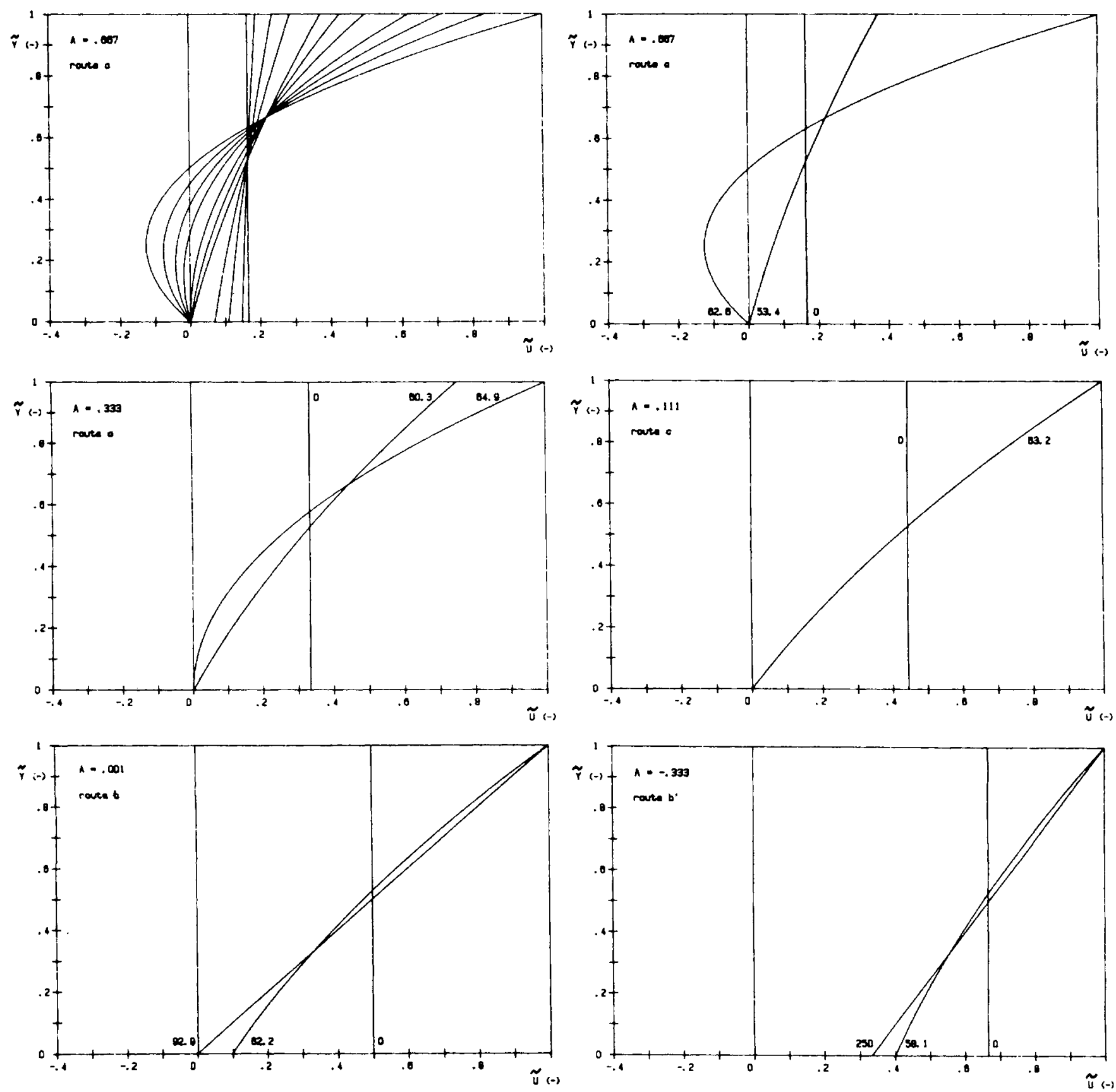

Fig. 19. Velocity profiles at the beginning of the extruder $(\tilde{x}=0)$, the first slip-stick transition point ( $\left.\tilde{x}_{1}\right)$, and (if present) the second slip-stick transition point $\left(\tilde{x}_{2}\right)$ for different routes and consequently, different throughputs. The first graph shows a developing velocity profile in more detail.

treme effects can be reached if slip is completely prevented at the barrel, for instance, by grooves or pins in the barrel wall as in Boundary Layer Slip, above. Of course there is only one slipstick transition point now and, as in Slip at Both Boundaries, above, this point is never reached for high throughputs $(-1<A \leqslant 0)$.

Because the starting conditions for situation 3 and 4 from Fig. 16 are changed, the expressions for pressure and velocity profile are also changed (compare with Eqs 33, 34, 35, and 36):

3. $\tilde{X}=0, \tilde{P}=\tilde{P}_{\text {o }}$

$$
\begin{gathered}
\tilde{P}=\left(\tilde{P}_{o}-s(1+A) / \mu_{o}\right) e^{-s 3 / 2 \mu_{o} \tilde{x}} \\
+s(1+A) / \mu_{o}
\end{gathered}
$$

$$
\begin{aligned}
\tilde{u}=1 & +(1+A)(\tilde{y}-1) \\
& +s\left(\mu_{o} \tilde{P}_{o}-s(1+A)\right) \\
& \cdot\left(\tilde{y}-3 / 4 \tilde{y}^{2}-1 / 4\right) e^{-s 3 / 2 \mu_{o} \tilde{x}}
\end{aligned}
$$

4. $\tilde{x}=\tilde{x}_{1}, \tilde{P}=\tilde{P}_{1}$

$$
\begin{aligned}
& \tilde{P}=\tilde{P}_{1}+6 A\left(\tilde{x}-\tilde{x}_{1}\right) \\
& \tilde{u}=\tilde{y}+3 A\left(\tilde{y}^{2}-\tilde{y}\right)
\end{aligned}
$$

$3 \rightarrow 4$, the slip-stick transition point:

$$
\begin{aligned}
& \tilde{x}_{1}=-2 s \ln \left(4 A /\left((1+A)-s \mu_{o} \tilde{P}_{o}\right)\right) /\left(3 \mu_{o}\right) \\
& \tilde{P}_{1}=s(1-3 A) / \mu_{o}
\end{aligned}
$$


Finally, the expression for the pumping efficiency now reads

$$
\eta_{p}=1 / 2(1-A)\left(\tilde{P}_{e}-\tilde{P}_{o}\right)\left(\int 3+\int 4\right)
$$

with

$$
\begin{gathered}
\int_{3}=\left(\mu_{o} \tilde{P}_{o}-s(1+A)\right)\left(e^{-s 3 / 2 \mu_{o} \tilde{x}_{1}}-1\right) / \\
\cdot\left(3 \mu_{o}\right)+(1+A) \tilde{x}_{1}
\end{gathered}
$$

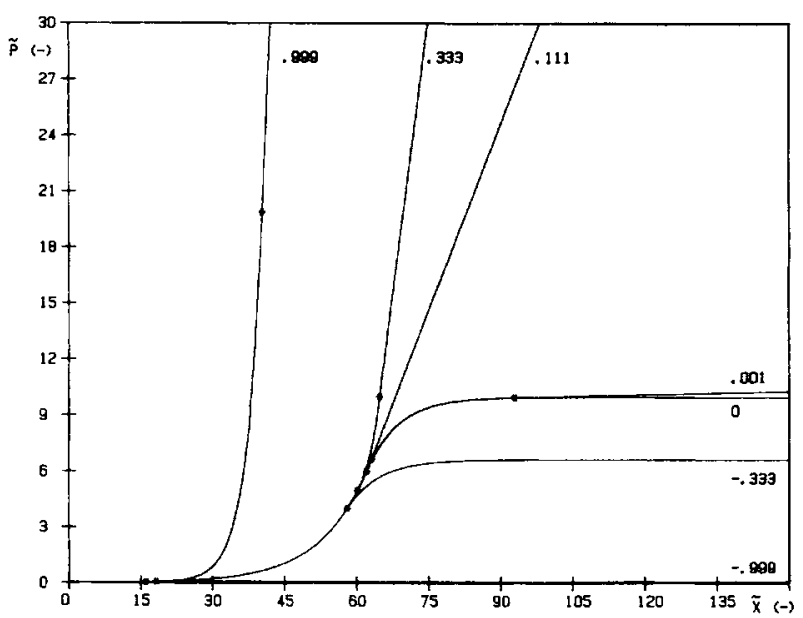

Fig. 20. Pressure profile, parameter throughput, A. Slip stick transition points are denoted with *.

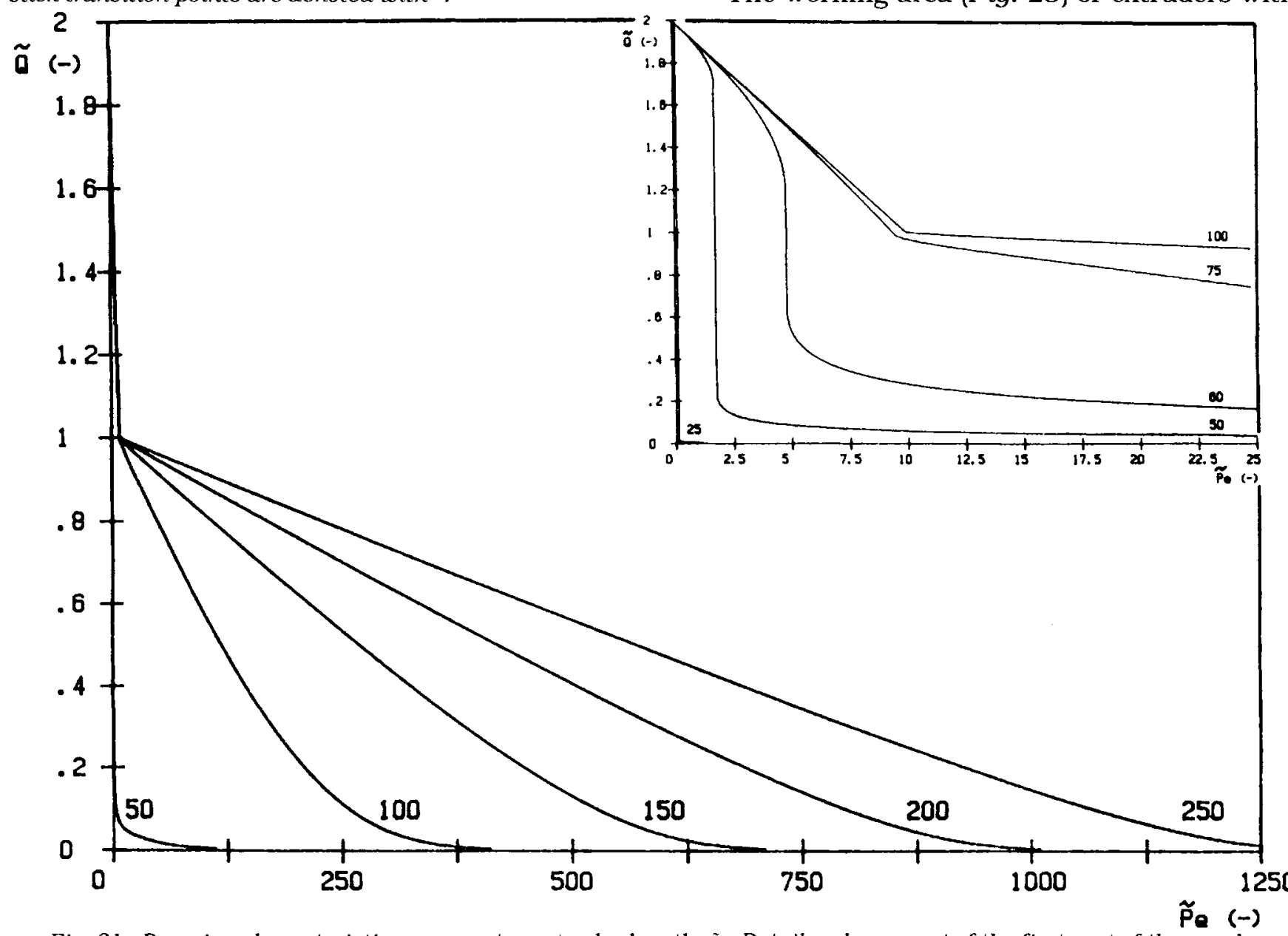

Fig. 21. Pumping characteristics, parameter extruder length, $\tilde{x}_{e}$. Detail: enlargement of the first part of the graph.
For all equations, $s=1$.

For higher outputs $(-1<A<\sim 1 / 3)$, the slip velocities are positive. Small outputs $(\sim 1 / 3<A<$ 1), however, imply negative slip velocities. Therefore, for small outputs the shear stresses near the screw must have the opposite direction to those of large outputs. Consequently, the slip boundary condition changes from $\tau=-\mu_{0} P_{o}$ into $\tau=\mu_{o} P_{o}$. For both cases, Eqs 49 through 55 are valid. For small outputs, however, the sign of $s$ changes into -1 .

The condition for the change of sign is indicated as $A=\sim 1 / 3$. Around the value $A=1 / 3$, a region exists where no slip occurs at all. The size of this region depends on the values of $\mu_{o}$ and $\tilde{P}_{\mathrm{o}}$ and can be found from Eq. 53 supposing $\tilde{\boldsymbol{x}}_{1}=0$ and $s$ either 1 or -1 :

$$
1 / 3-\mu_{o} \tilde{P}_{o} \leqslant A_{s} \leqslant 1 / 3+\mu_{o} \tilde{P}_{o} .
$$

In accordance with Fig. 8 , the shear rate and, therefore, the shear stress at the screw are zero for $a=1 / 3$. Consequently, no stress is needed to maintain the stick condition. Small deviations for $d u / d y$ around $A=1 / 3$ are possible without slip as long as the shear stress is smaller than $\mu_{0} P_{0}$.

The working area (Fig. 23) of extruders with 


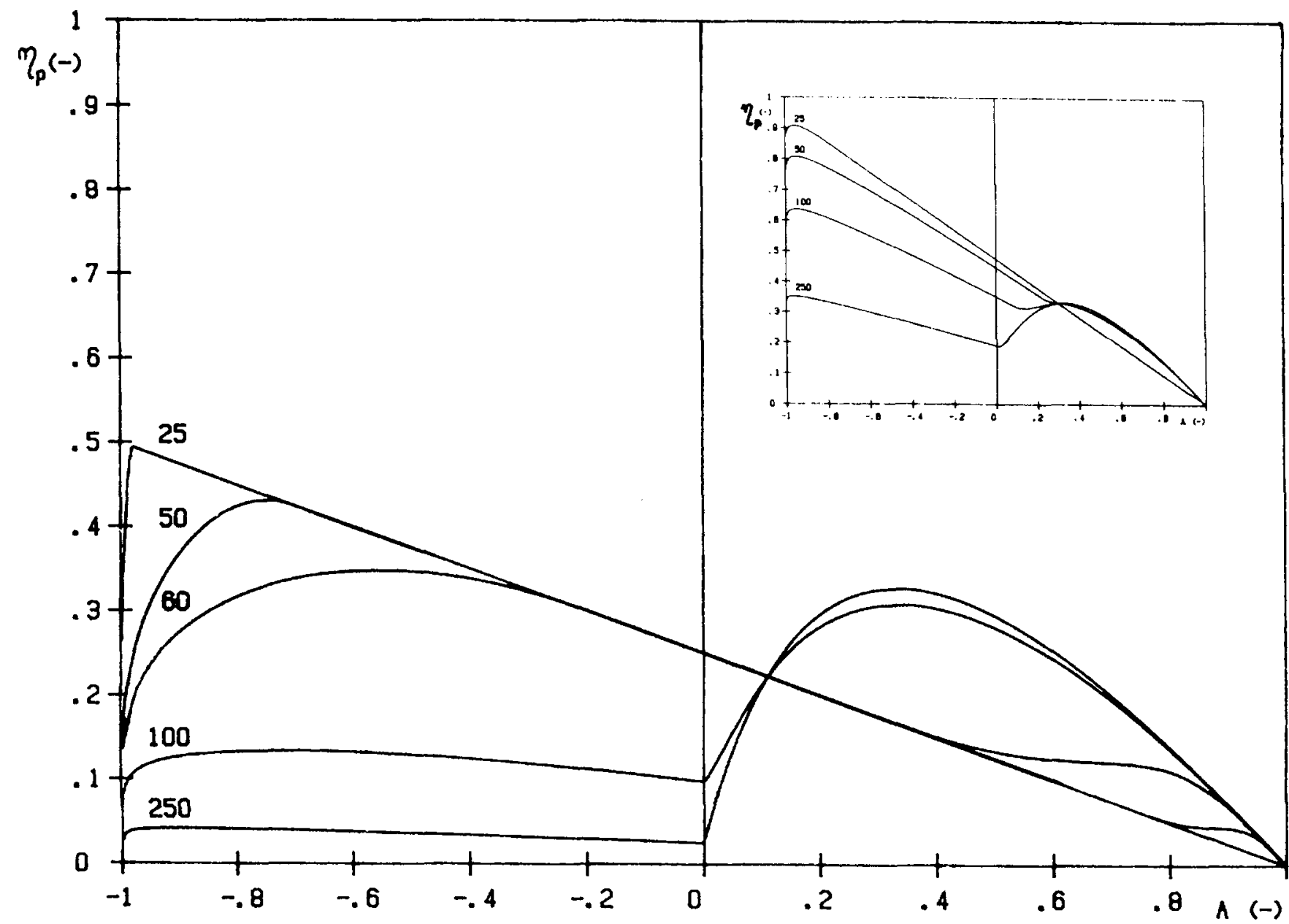

Fig. 22. Pumping efficiency us. throughput, parameter extruder length $\tilde{x}_{e}$. Detail: the same graph for $\mu_{o}=0.01$.

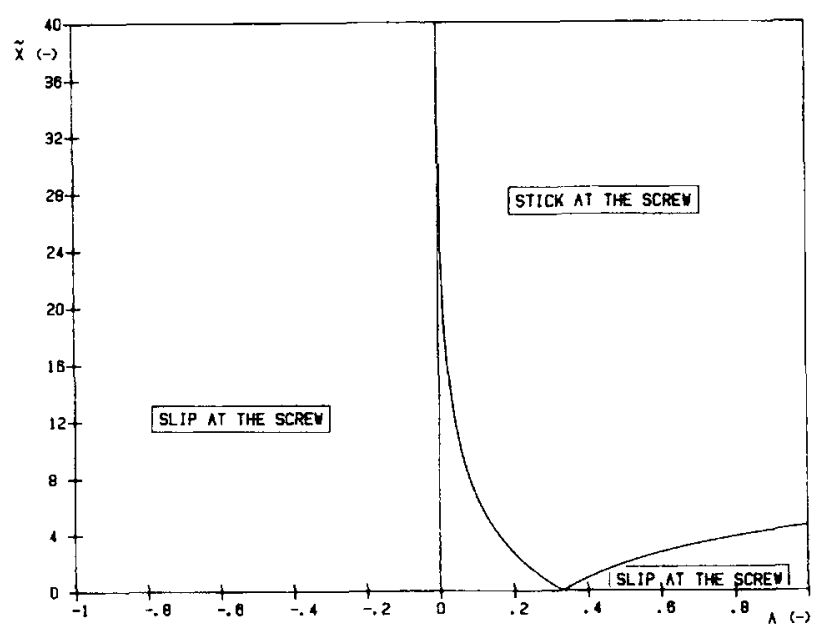

Fig. 23. Working area for extruders with friction slip at the screw only $\left(H=0.006 \mathrm{~m}, \eta=1000 \mathrm{~Pa} . \mathrm{s}, P_{o}=1000 \mathrm{~Pa}\right.$, $U=0.5 \mathrm{~m} / \mathrm{s}, \mu_{0}=0.11$.

one side friction slip can easily be constructed with Eqs 53 and 56 and can be compared with the two side slip case of Fig. 18. In Figs. $24 b$ and $24 c$, the lines of transition points are given in more detail with parameter $\mu_{o}$ and $P_{o}$ respectively. With increasing friction and starting pressure, the transition point gets closer to the
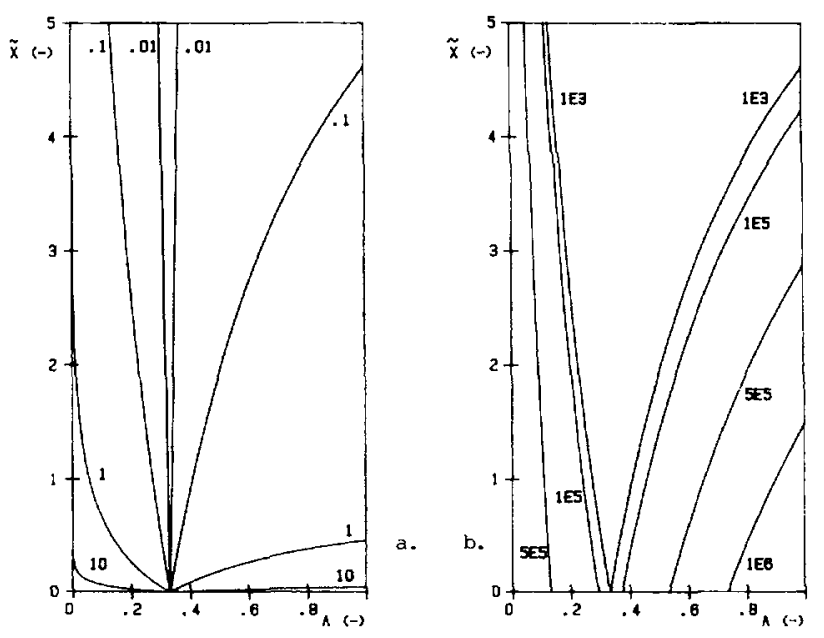

Fig. 24. Details of Fig. 23: a. parameter, friction coefficient at the screw, $\mu_{0} ; b$. parameter initial pressure, $P_{0}$ $(\mathrm{Pa})$.

beginning of the extruder and the area of no slip becomes larger.

Some developing velocity profiles are plotted in Fig. 25 for different values of the throughput $A$. The corresponding pressure profiles are shown in Fig. 26. They also show the difference between small and large outputs with or with- 

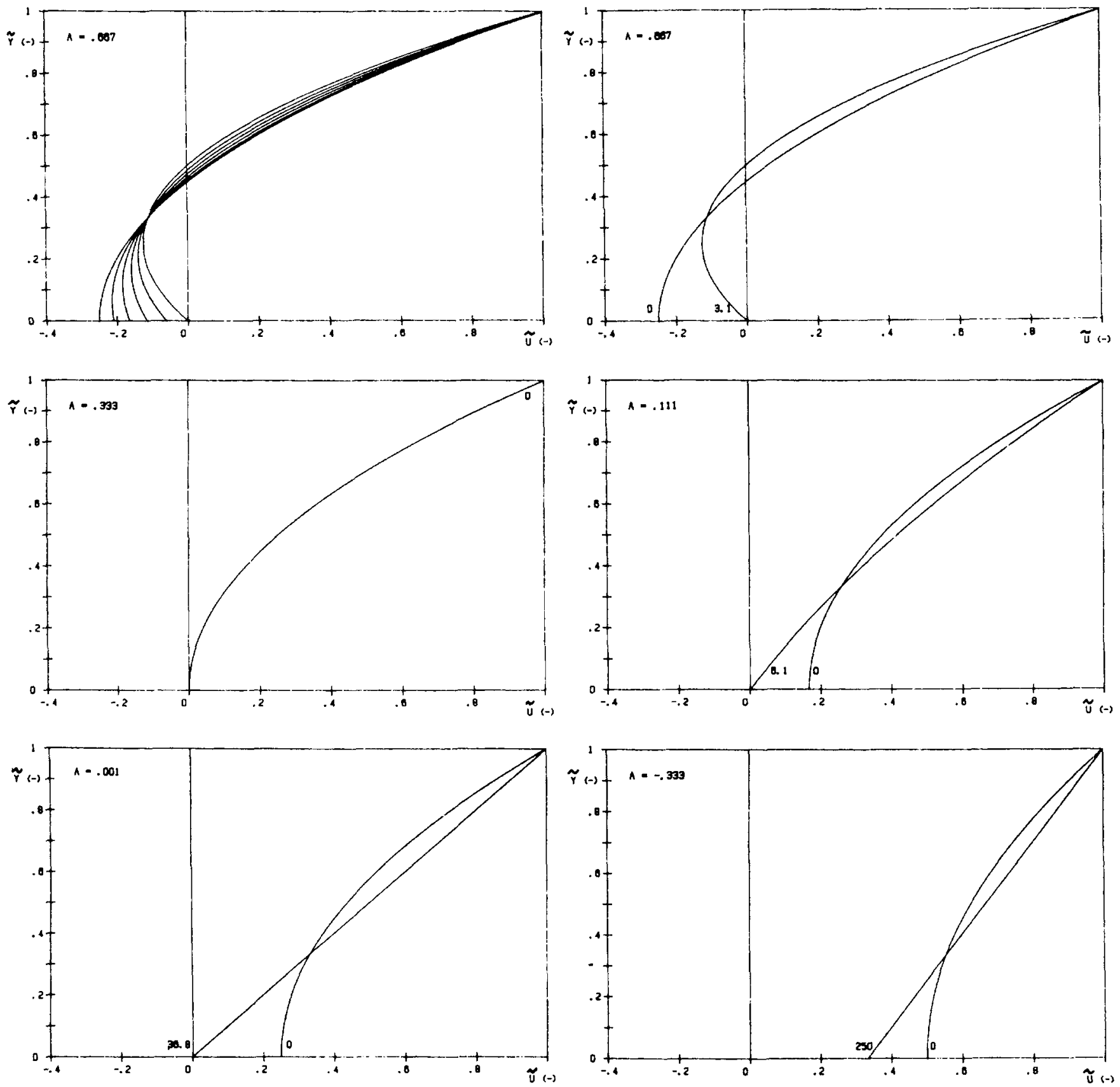

Fig. 25. Velocity profiles, at the beginning of the extruder $(\tilde{x}=0)$ and (if present) at the slip-stick transition point $\left(\tilde{x}_{1}\right)$ for different throughputs. The first graph shows a developing velocity profile in more detail.

out the slip-stick transition. In Figs. 27 and 28, the pumping characteristics and efficiencies are given for different extruder lengths, $\tilde{\boldsymbol{x}}_{e}$. The pumping efficiency is again high for the high output range (with small extruder lengths) and resembling the efficiency in the case of stick for low outputs (with large extruder lengths); compare Figs. 9, 12, 15, and 22.

\section{DISCUSSION}

After numerous equations yielding as many graphs, it has become clear that slip boundary conditions can change the extrusion behavior significantly. Nevertheless, the models presented can only give a rough idea of what can happen in practice. The two-dimensional Newtonian isothermal treatment with constant slip parameters while not very realistic, is straightforward and simple. The possible influence of slip on the pumping characteristics of extruders, the efficiency, velocity profiles, and consequently, the residence time distribution has become qualitatively clear. Molecular models of slip have been ignored; moreover, measurements of slip and the general question of which slip condition is valid for which material have not been discussed.

Many authors have tried to perform measurements with transparent flat bore and grooved capillaries $(1,2,8,9,31,32)$, with colored slices $(3,4,5)$, with capillaries with different diame- 
ters $(12,13,33)$, in rotational viscosimeters $(34$, $35)$, and in extruders $(23,25,36,37)$. Quantitative measurement of slip proves to be rather difficult (38). Also, in extruders it is not immediately obvious whether slip occurs. This can be seen from Fig. 29, which gives a combination of an extruder with a die, resulting in a throughput-screw speed plot for more or less realistic values of boundary layer slip coefficients, coefficients of friction, and viscosity. For compari-

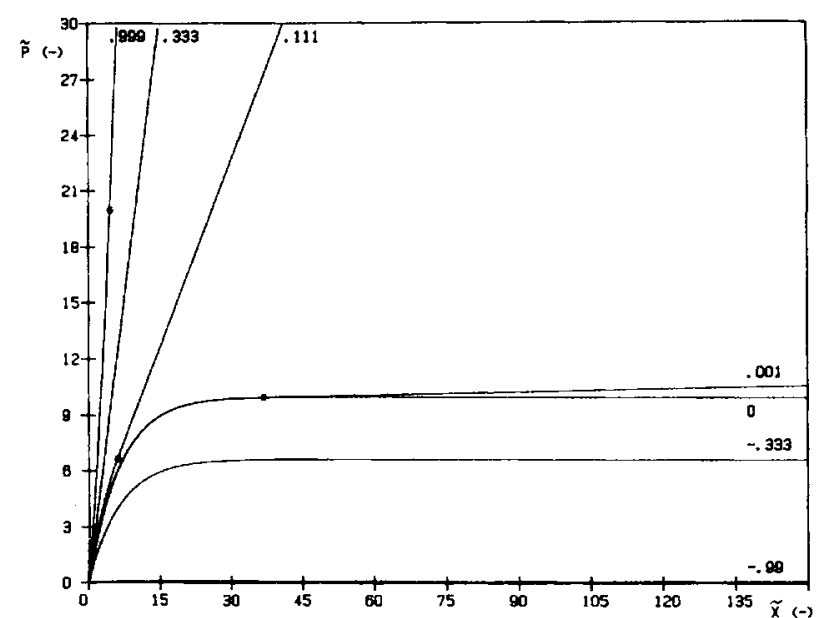

Fig. 26. Pressure profiles, parameter throughput, A. Slipstick transition points are denoted with *. son, the nonlinear power-law lines are also plotted. It is clear that there are differences, but they only show up in a different slope of the $Q$ $-N$ plot. Given the simplicity of the models, a change in slope alone is not enough to differentiate between the different slip phenomena. To achieve such differentiation, the total pumping characteristics and efficiencies as given in the previous figures must be measured.

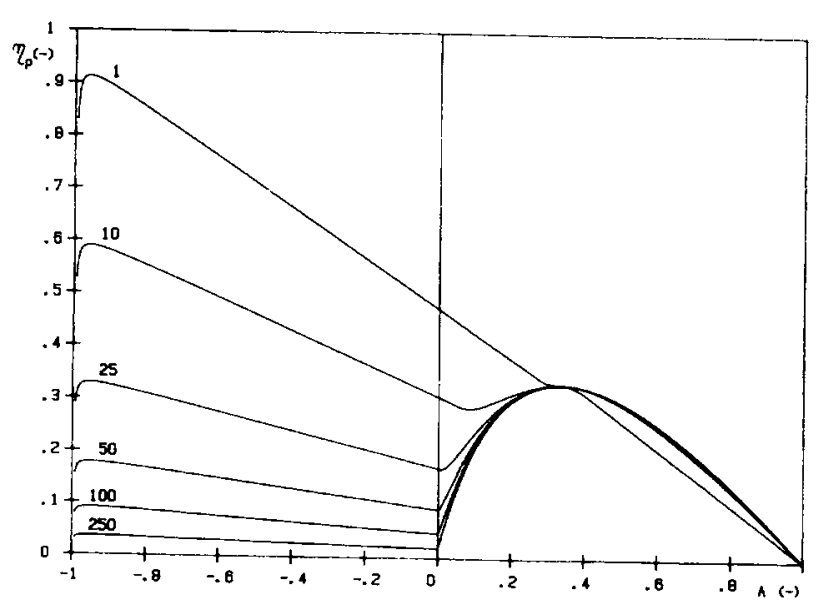

Fig. 28. Pumping efficiency vs. throughput, parameter extruder length, $\tilde{x}_{e}$.

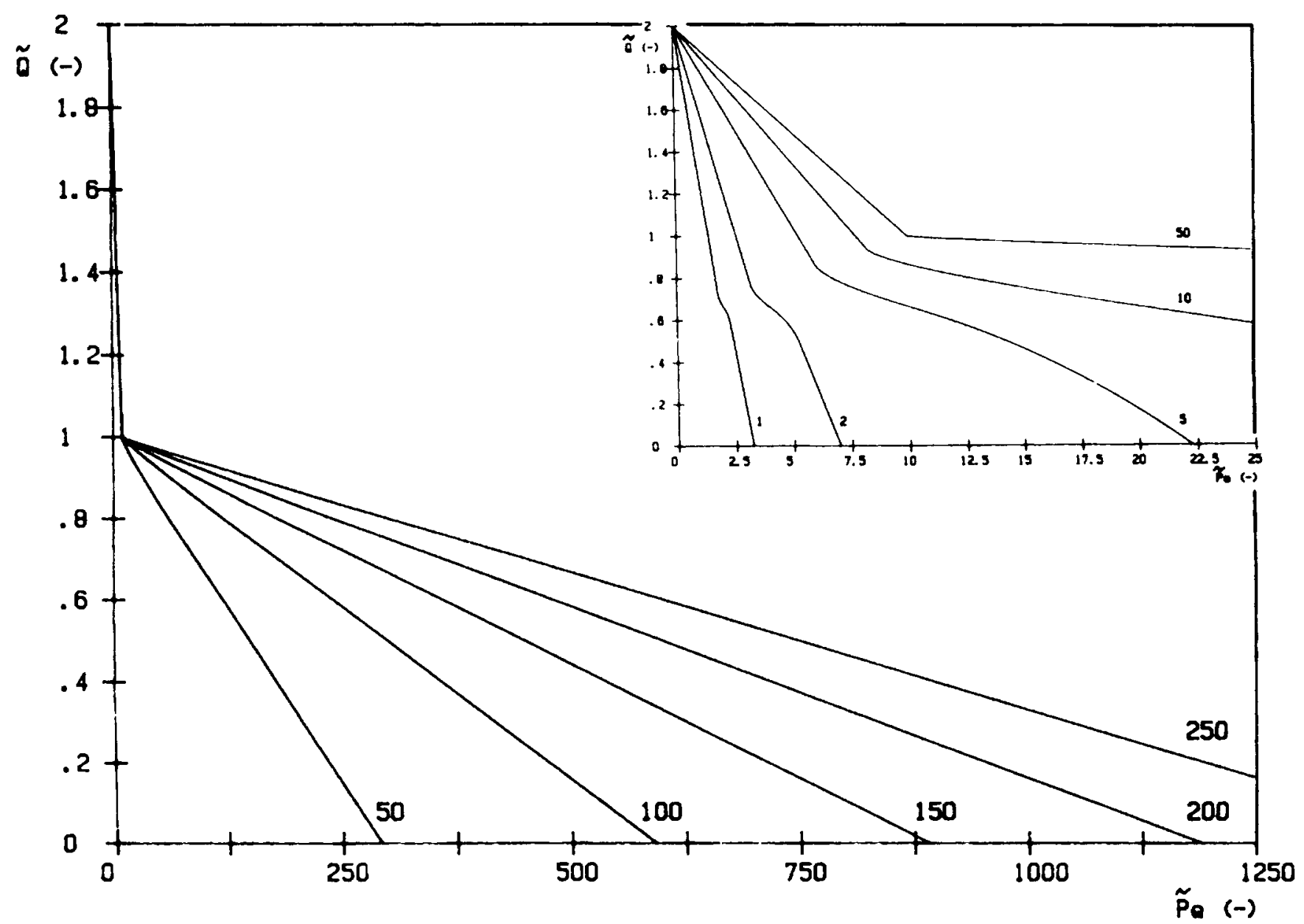

Fig. 27. Pumping characteristics, parameter extruder length, $\tilde{x}_{e}$. Detail: enlargement of the first part of the graph. 

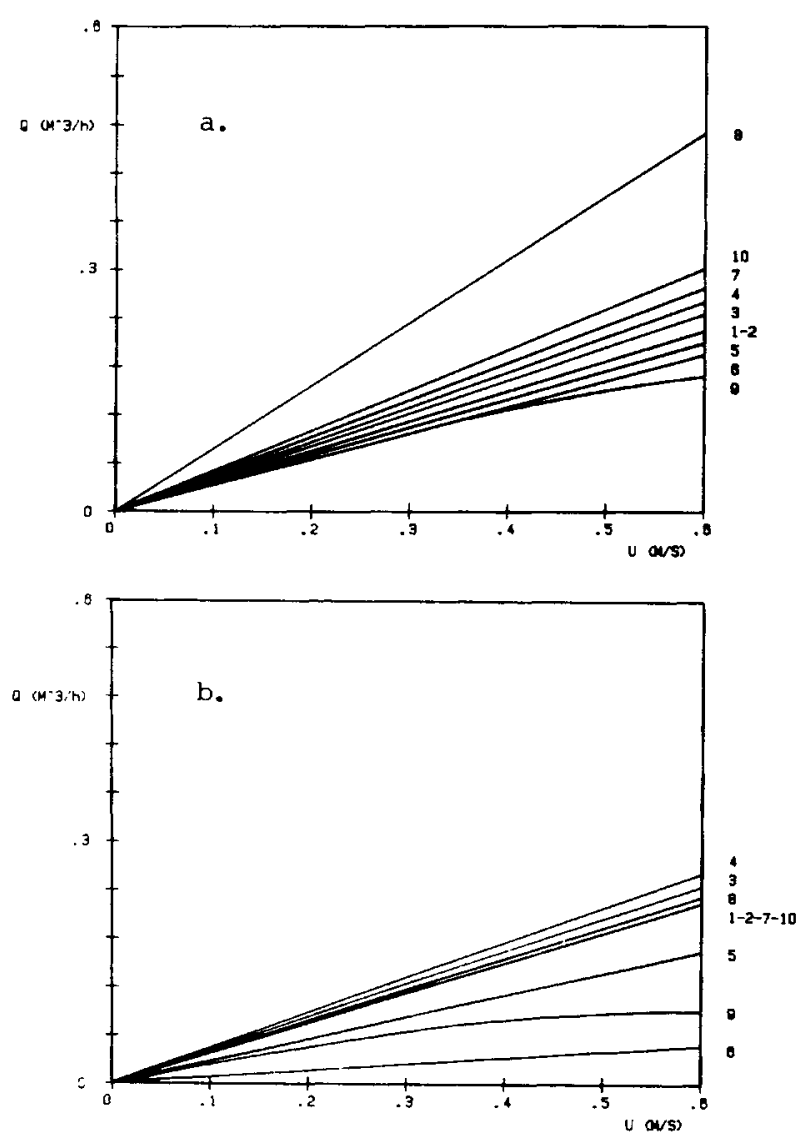

Fig. 29. Throughput vs. screw speed: a. the same bound ary condition for extruder and capillary; $b$. stick boundary condition for the capillary. Extruder: $L=2 \mathrm{~m}, H=$ $0.006 \mathrm{~m}, W=0.05 \mathrm{~m}$. Capillary: $L=0.1 \mathrm{~m}, R=0.004 \mathrm{~m}$. 1. Stick. Newtonian $\eta=1000$ Pa. 2. Stick, Newtonian $\eta=$ $500 \mathrm{~Pa}$. 3. Stick, power law, $\eta_{o}=2660, n=0.75$. 4. Stick, power law, $\eta_{o}=7070, n=0.5$. 5. Boundary layer slip, $B=$ 5. 6. Boundary layer slip, $B=0.5$. 7. Boundary layer slip (in the extruder at the screw only), $B=5.8$. Boundary layer slip (in the extruder at the screw only), $B=0.5$. 9 . Friction slip, $\mu_{h}=0.2, \mu_{0}=0.18, \mu=0.2, P_{o}=1000 \mathrm{~Pa} .10$. Friction slip (in the extruder at the screw only), $\mu_{o}=0.2$, $\mu=0.2, P_{o}=1000 \mathrm{~Pa}$.

\section{NOMENCLATURE}

$a=$ throttle ratio $(-)$.

$A=$ measure for throughput, like $a(-)$.

$A_{s}=$ ditto, where no slip occurs (friction model) $(-)$.

$B=$ dimensionless slip coefficient in a capillary $(-)$.

$B_{o}=$ dimensionless slip coefficient at the screw $(-)$.

$B_{h}=$ dimensionless slip coefficient at the barrel $(-)$.

$c=$ slip coefficient in a capillary $=\eta_{r} / \delta$ (Pa.s $/ \mathrm{m})$.

$c_{o}=$ slip coefficient at the screw $=\eta_{o} / \delta_{o}$ (Pa.s/m).

$c_{h}=$ slip coefficient at the barrel $=\eta_{h} / \delta_{h}$ (Pa.s/m).

$H=$ height of an extruder channel (m).

$K=$ dimensionless pressure gradient $(-)$.

$L=$ length of a capillary $(\mathrm{m})$.

$P=$ pressure $(\mathrm{Pa})$.
$P_{o}=$ pressure at the beginning of an extruder or at the end of a capillary $(\mathrm{Pa})$.

$P_{1}=$ pressure at the first transition point $(\mathrm{Pa})$.

$P_{2}=$ pressure at the second transition point $(\mathrm{Pa})$.

$P_{e}=$ pressure at the end of an extruder $(\mathrm{Pa})$.

$P_{L}=$ pressure at the beginning of a capillary (Pa).

$Q=$ throughput in extruders (two-dimensional flow) $\left(\mathrm{m}^{2} / \mathrm{s}\right)$.

$r=$ radial distance $(\mathrm{m})$.

$R=$ radius of a capillary $(\mathrm{m})$.

$u=$ axial velocity in an extruder $(\mathrm{m} / \mathrm{s})$.

$u_{o}=$ velocity at the screw $(\mathrm{m} / \mathrm{s})$.

$u_{h}=$ velocity at the barrel $(\mathrm{m} / \mathrm{s})$.

$u_{\mathrm{s}}=$ slip velocity at the barrel $(\mathrm{m} / \mathrm{s})$.

$U=$ barrel velocity $(\mathrm{m} / \mathrm{s})$.

$w=$ axial velocity in a capillary $(\mathrm{m} / \mathrm{s})$.

$w_{r}=$ slip velocity in a capillary $(\mathrm{m} / \mathrm{s})$.

$\bar{w}=$ average velocity in a capillary $(\mathrm{m} / \mathrm{s})$.

$W=$ width of an extruder channel (m).

$x=$ axial distance in an extruder $(\mathrm{m})$.

$x_{1}=$ first slip-stick transition point in an extru$\operatorname{der}(\mathrm{m})$.

$x_{2}=$ second slip-stick transition point in an extruder $(\mathrm{m})$.

$y=$ radial distance in an extruder $(\mathrm{m})$.

$Z=$ axial distance in a capillary $(\mathrm{m})$.

$z_{1}=$ stick-slip transition point in a capillary (m).

Dimensionless parameters are denoted with $a \sim$.

$\dot{\gamma}=$ shear rate $\left(\mathrm{s}^{-1}\right)$.

$\eta=$ viscosity of the bulk material (Pa.s).

$\eta_{0}=$ viscosity of the boundary layer at the screw (Pa.s).

$\eta_{h}=$ viscosity of the boundary layer at the barrel (Pa.s).

$\eta_{r}=$ viscosity of the boundary layer in a capillary (Pa.s).

$\eta_{p}=$ pumping efficiency (-).

$\delta=$ boundary layer thickness in a capillary (m).

$\delta_{o}=$ boundary layer thickness at the screw (m).

$\delta_{h}=$ boundary layer thickness at the barrel (m).

$\mu=$ coefficient of friction in a capillary $(-)$.

$\mu_{o}=$ coefficient of friction at the screw $(-)$.

$\mu_{h}=$ coefficient of friction at the barrel $(-)$.

$\tau=$ shear stress $(\mathrm{Pa})$.

\section{REFERENCES}

1. H. Offermann, thesis, TH Aachen (1972).

2. H. Offermann, Angew. Makromol. Chem., 47, 217 (1975).

3. G. Mennig, Rheol. Acta, 15, 199 (1976).

4. G. Mennig. J. Macromol. Sci.-Phys., 14, 231 (1977).

5. R.-P. Hegler and G. Mennig, Rheol. Acta, 20, 553 (1981).

6. W. Knappe and E. Krümbock, Proc. IX Intl. Congress on Rheology, Mexico (1984).

7. J. L. den Otter and J. L. S. Wales, Ann. Conf. of the Dutch Rheological Sociëty, Wageningen (1981).

8. E. Uhland, thesis, IKT Stuttgart (1978).

9. E. Uhland, Rheol. Acta, 18, 1 (1979).

10. J. L. den Otter, Rheol. Acta, 14, 329 (1975).

11. F. Buschhaus, internal report, IKV Aachen (1983). 
12. M. Mooney, J. Rheology, 2, 210 (1931).

13. H. Umstätter, Einführung in die Viscosimetrie und Rheometrie, 38-46, Springer Verlag, Berlin (1952).

14. R. Holslag, Seminar on Extrusion in the Food industry, Wageningen (1980).

15. J. F. Ingen Housz, ibid., Wageningen (1980).

16. J. F. Ingen Housz, $Z F L, 2,89$ (1983).

17. E. Uhland, Rheol. Acta, 15, 30 (1976).

18. W. H. Darnell and E. A. J. Mol, SPE J., 12, 4 (1956).

19. Z. Tadmor, et al., Technical Papers, 29th Annual Technical Conference, Society of Plastics Engineers, 136 , Washington (1971).

20. Z. Tadmor, et al., ibid., 141 (1971).

21. Z. Tadmor and E. Broyer, Polym. Eng. Sci., 12, 378 (1972).

22. Z. Tadmor, Polym. Eng. Sci., 6, 185 (1966).

23. D. Schlegel, Chem.-Ing.-Tech., 49, 985 (1977).

24. R. A. Worth and H. A. A. Helmy, Plast. Rubber: Process., 2, 3 (1977)

25. R. A. Worth, et al., Polym. Eng. Sci., 17, 257 (1977).

26. G. Mennig, Kunststoffe, 70, 254 (1980).
27. G. Mennig, Kunststoffe, 74, 296 (1984).

28. P. Perwadtschuk, et al., Plaste Kautschuk, 29, 237 (1982).

29. Z. Tadmor, et al., SPE ANTEC Tech. Papers, 25, 193 (1979).

30. P. Hold, et al., SPE ANTEC Tech. Papers, 25, 205 (1979).

31. J. J. Benbow and P. Lamb, SPE Trans., 7 (January 1963).

32. J. L. den Otter, et al., Rheol. Acta, 6, 205 (1967).

33. W. Gleissle and E. Windhab, Experiments in Fluids, 3, 177 (1985).

34. M. Mooney, in "Rheology" by F. Eirich, Bd. II, 197-200 and 217-218, Academic Press Inc., New York (1958).

35. R. Rautenbach and D. Schlegel, Chem.-Ing.-Tech., 41, 369 (1969).

36. G. Mennig, Kunststoffe, 71, 359 (1981)

37. G. Mennig and N. Subramanian, Polym. Eng. Sci., 22, 747 (1982)

38. R. Berger, Plaste Kautschuk, 2, 113 (1972). 